\title{
Estimating permeability from quasi-static deformation: Temporal variations and arrival time inversion
}

\author{
D. W. Vasco* \\ Earth Sciences Division, Berkeley Laboratory, Berkeley, CA 94720
}

\begin{abstract}
Alessandro Ferretti*
Tele-Rilevamento Europa, T.R.E. s.r.l., Via Vittoria Colonna, 7-20149 Milano, Italy

\section{Fabrizio Novali*}

Tele-Rilevamento Europa, T.R.E. s.r.l., Via Vittoria Colonna, 7-20149 Milano, Italy
\end{abstract}

(January 23, 2008)

\begin{abstract}
Transient pressure variations within a reservoir can be treated as a propagating front and analyzed using an asymptotic formulation. From this perspective one can define a pressure 'arrival time' and formulate solutions along trajectories, in the manner of ray theory. We combine this methodology and a technique for mapping overburden deformation into reservoir volume change as a means to estimate reservoir flow properties, such as permeability. Given the entire 'travel time' or phase field, obtained from the deformation data, we can construct the trajectories directly, there-by linearizing the inverse problem. A numerical study indicates that, using this approach, we can infer large-scale variations in flow properties. In an application to Interferometric Synthetic Aperture (InSAR) observations associated with a $\mathrm{CO}_{2}$ injection at the Krechba field, Algeria, we image pressure propagation to the northwest. An inversion for flow properties indicates a linear trend of high permeability.
\end{abstract}


The high permeability correlates with a northwest trending fault on the flank of the anticline which defines the field.

\section{INTRODUCTION}

In many activities, such as petroleum production, geothermal energy extraction, $\mathrm{CO}_{2}$ disposal, and groundwater utilization, knowledge of the structures controlling fluid flow at depth is critical. Significant effort has been expended to better understand fluid flow in the subsurface using flow and transport and time-lapse geophysical observations. For example, new instrumentation and reliable sensors have advanced the state-of-the-art in flow and transport monitoring, allowing for crosswell pressure tomography, multi-level samplers, and long term downhole pressure measurements (Freyberg 1986, Butler et al. 1999, Hsieh et al. 1985, Paillet 1993, Cook 1995, Masumoto et al. 1995, Karasaki et al. 2000, Yeh and Liu 2000, Vesselinov et al, 2001, Vasco and Karasaki 2001, Gringarten et al. 2003). There have been numerous efforts to use geophysical observations, particularly time-lapse data, to monitor fluid flow at depth. Much of this work has concentrated on seismic time-lapse, or 4D, amplitude data from oil and gas reservoirs (Barr 1973, Greaves and Fulp 1987, Nur 1989, Eastwood et al. 1994, Lee et al. 1995, Lazaratos and Marion 1997, Huang et al. 1998, Johnson et al. 1998, Burkhart et al. 2000, Behrens et al. 2002, Arts et al. 2004). Improved understanding of fluid flow in the shallow sub-surface has resulted from shallow and crosswell time-lapse electromagnetic surveys (Brewster and Annan 1994, Wilt et al. 1995, Barker and Moore 1998, Binley et al. 2001, Hoversten et al. 2003). Geodetic data provide still another set of observations related to fluid flow in the subsurface (Castle et al. 1969, Evans et al. 1982, Vasco et al. 1988, Palmer 1990, Dussealt et al. 1993, Bruno and Bilak 1994, Chilingarian et al. 1994, Castillo et al. 1997, Massonnet et al. 1997, Vasco et al. 1998, Wright 1998, Wright et al. 1998, Mossop and Segall 1999, Stancliffe and van der Kooij 2001, Vasco et al. 2001, Du 
and Olson 2001, Hoffmann et al. 2001, Vasco and Ferretti 2005).

In spite of these efforts, our fundamental understanding of fluid flow within the Earth is hampered by a number of factors. For example, there is often limited data coverage, particularly with regard to flow and transport measurements. Similarly, the temporal sampling can be sparse, particularly with regard to time-lapse geophysics surveys which can be years apart, missing important transient phenomena. Frequently, the scale of investigation is small, of the order of a few meters, and the results might not scale up to the kilometer range, where faults and fracture zones control flow. In addition, it is often difficult to relate geophysical attributes, like seismic amplitudes, to flow properties. There can be trade-offs between various sets of attributes such as between mechanical properties and fluid pressure estimates. Finally, in applications one typically requires knowledge of factors influencing sub-surface flow in a timely fashion in order to optimize or mitigate the activities.

In this study we employ a cost-effective technique for imaging fluid flow and estimating flow properties, such as permeability. We use time-varying measurements of deformation in the overburden as data. Such observations are of increasing importance for at least two reasons. First, there have been several developments in the past few years advancing the state-of-the-art in geodetic monitoring. For example, downhole tilt meters can provide dense time sampling of deformation at a wide range of depths, from the surface to several thousand meters (Wright 1998, Wright et al. 1998, Du et al. 2005). Space-based techniques, such as Interferometric Synthetic Aperture Radar (InSAR), can sample surface deformation with a spatial resolution (pixel size) of meters to tens of meters and an accuracy of the order of a centimeter to a few millimeters (Massonnet and Feigl 1998, Burgmann et al. 2000, Ferretti et al. 2000, 2001). InSAR observations may be used to image fluid-induced deformation over regions tens to hundreds of square kilometers in extent (Fielding et al. 1998, Galloway et al. 1998, Amelung et al. 1999, Hoffmann et al. 2001, Schmidt and Burgmann 2003, Colesanti et al. 2003, Vasco and Ferretti 2005). Second, recent developments 
in the processing of time-lapse seismic data allow for estimates of deformation in the overburden and within the reservoir (Guilbot and Smith 2002, Landro and Stammeijer 2004, Tura et al. 2005, Hatchell and Bourne 2005, Barkved and Kristiansen 2005, Hall 2006, Roste et al. 2006, Rickett et al. 2007, Staples et al. 2007, Hawkins et al. 2007). Such estimates offer high resolution, three-dimensional sampling of deformation over a producing reservoir. Based upon a geomechanical model the deformation can be mapped into pressure changes within the reservoir (Vasco et al. 2000a, Du et al. 2005, Vasco and Ferretti 2005, Hodgson et al. 2007).

It is possible to use the estimated pressure changes directly to infer flow properties, such as permeability (Vasco et al. 2001, Vasco 2004a, Vasco and Ferretti 2005). Here we take an alternative approach, based upon the idea of a diffusive travel time associated with a propagating pressure transient (Vasco et al. 2000b, Vasco 2004b). Though the approach requires adequate sampling of the pressure field in time, it is relatively insensitive to the geomechanical properties within the reservoir and their heterogeneity. Furthermore, the approach shares the robustness of arrival time tomography and actually leads to a linear inverse problem for flow properties. Thus, it is possible to map deformation measurements into reservoir permeability in a linear fashion.

\section{METHODOLOGY}

Before we discuss the approach in detail we outline the major steps of our inversion methodology. The first step entails inverting measurements of overburden deformation in order to estimate volume change within the reservoir. Next, the volume change is mapped into fluid pressure change induced by the production or injection. Finally, the fluid pressure changes $p(\mathbf{x}, t)$ are used to estimate flow properties in the reservoir using a technique similar to travel time tomography applied to a disturbance which

evolves according to a diffusion equation. Because this latter step is unfamiliar to 
most readers, we shall discuss it in more detail in this section. The first two steps have been described in earlier papers (Vasco et al. 1988, Vasco et al. 1998, Mossop and Segall 1999, Vasco et al. 2000a, Du and Olson 2001, Vasco et al. 2001, Vasco et al. 2002, Vasco and Ferretti 2005) so we shall only outline them briefly in the Appendix.

\section{A Diffusion Equation for Fluid Pressure Variations}

We are interested in the propagation of pressure changes within a reservoir. The pressure changes are caused by production and/or injection from wells located within the reservoir itself. The fluid volume changes are determined by the flow rate out of, or into, the reservoir, denoted here by the source-time function $q(\mathbf{x}, t)$. The reservoir will deform due to the fluid volume changes and that deformation will lead to displacements in the overlying material.

Our starting point is the set of partial differential equations describing the flow of an aqueous phase and a non-aqueous phase (Peaceman, 1977)

$$
\begin{aligned}
& \nabla \cdot\left[\frac{\rho_{w} K(\mathbf{x}) k_{r w}}{\mu_{w}} \nabla\left(p_{w}(\mathbf{x}, t)-\rho_{w} g z\right)\right]=\frac{\partial\left(\phi \rho_{w} S_{w}\right)}{\partial t} \\
& \nabla \cdot\left[\frac{\rho_{n} K(\mathbf{x}) k_{r n}}{\mu_{n}} \nabla\left(p_{n}(\mathbf{x}, t)-\rho_{n} g z\right)\right]=\frac{\partial\left(\phi \rho_{n} S_{n}\right)}{\partial t}+q
\end{aligned}
$$

where $S_{w}$ and $S_{n}$ denote the saturations of the aqueous and non-aqueous phases respectively and $q$ denotes the injection rate of the non-aqueous phase. The relative permeabilities of the aqueous and non-aqueous phases are represented by $k_{r w}$ and $k_{r n}$ while the absolute permeability is given by $K(\mathbf{x})$. The respective densities are $\rho_{w}$ and $\rho_{n}$, the gravitational constant is $g$ and the porosity is $\phi(\mathbf{x})$. The pressure associated with the aqueous phase is $p_{w}(\mathbf{x}, t)$ while the pressure for the non-aqueous phase is $p_{n}(\mathbf{x}, t)$, the viscosities are $\mu_{w}$ and $\mu_{n}$. The above equations are coupled because the saturations are assumed to sum to unity

$$
S_{w}+S_{n}=1
$$


Typically, in modeling $\mathrm{CO}_{2}$-brine systems it is assumed that capillary effects are negligible (Pruess 2004, Obi and Blunt 2006) and hence $p_{w}=p_{n}=p$, where $p$ denotes the common fluid pressure. Adding equations (1) together and assuming that the pressure in the aqueous and non-aqueous phases are equal results in

$$
\begin{gathered}
\nabla \cdot K\left[\kappa \nabla p-\mathbf{g}_{f}\right]=\frac{\partial\left(\phi \sigma_{f}\right)}{\partial t}+q, \\
\kappa=\frac{\rho_{w} k_{r w}}{\mu_{w}}+\frac{\rho_{n} k_{r n}}{\mu_{n}}, \\
\mathbf{g}_{f}=\frac{\rho_{w} k_{r w}}{\mu_{w}} \nabla\left(\rho_{w} g z\right)+\frac{\rho_{n} k_{r n}}{\mu_{n}} \nabla\left(\rho_{n} g z\right),
\end{gathered}
$$

and

$$
\begin{aligned}
& \sigma_{f}=\rho_{w} S_{w}+\rho_{n} S_{n} \\
& =\rho_{w}+\left(\rho_{n}-\rho_{w}\right) S_{n}
\end{aligned}
$$

where we have used the fact that the saturations sum to unity, equation (2). Equation (3) is a differential equation describing the evolution of the total fluid pressure in a heterogeneous medium due to a time-varying source $q(\mathbf{x}, t)$ (Rice and Cleary 1976).

Equation (3) is a single equation in several unknowns $\left[\rho_{w}, \rho_{n}, S_{w}, S_{n}, \phi\right.$ and $\left.p\right]$ and further assumptions are necessary in order to produce a useful expression. For example, we expand the time derivative in equation (3)

$$
\frac{\partial\left(\phi \sigma_{f}\right)}{\partial t}=\sigma_{f} \frac{\partial \phi}{\partial t}+\phi \frac{\partial \sigma_{f}}{\partial t}
$$

Using the relationship in equation (6), we can write the second partial derivative on the right-hand-side of (7) as

$$
\frac{\partial \sigma_{f}}{\partial t}=\frac{\partial \rho_{w}}{\partial t}+\frac{\partial\left(\Delta \rho S_{n}\right)}{\partial t}
$$

where $\Delta \rho=\rho_{n}-\rho_{w}$ is the density difference between water and $\mathrm{CO}_{2}$ under reservoir conditions. Typically, one can neglect the compressibility of water and treat the water 
density as a constant. The second term can be important when the compressibility of the non-aqueous phase is significant. For this reason, we shall maintain this term in our formulation. As we shall see, because we are interested in the 'arrival time' of the initial pressure change, this term will vanish because $S_{n}$ is insignificant at this time. Physically, this is due to the fact that the pressure change propagates much faster than the saturation change. Furthermore, at the pressures within the Krechba reservoir the density of the $\mathrm{CO}_{2}$ does not vary significantly and is close to that of water, between 0.85 and $0.90 \mathrm{gm} / \mathrm{cm}^{3}$.

When the solid constituents are incompressible, the compressibility of the rock is dominated by changes in pore volume and one typically has a relationship between fluid pressure $p$, the bulk or overburden pressure $p_{o}$, and porosity $\phi$, of the form

$$
p=F\left(p_{o}, \phi\right)
$$

The function $F$ is usually derived from laboratory measurements. In the fully general situation, porosity, permeability, and fluid density may be functions of fluid pressure and the overburden or confining pressure, leading to a non-linear generalization of the diffusion equation (3), (Audet and Fowler 1992, Wu and Pruess 2000).

In this study we adopt the theory of poroelasticity (Biot 1941), in which the relationship between the fluid pressure, the overburden pressure, and the change in porosity is linear:

$$
\phi-\phi_{0}=\frac{\alpha}{K_{u}} p_{o}+C_{e} p
$$

which involves the initial porosity $\phi_{0}$, the undrained bulk modulus $K_{u}$, and the constants, $\alpha$ and $C_{e}$ defined by Biot (1941). The variable $C_{e}(\mathbf{x})$ relates fluid pressure variations to changes in porosity. Differentiating equation (10) with respect to time gives

$$
\frac{\partial \phi}{\partial t}=C_{e} \frac{\partial p}{\partial t}+\frac{\alpha}{K_{u}} \frac{\partial p_{o}}{\partial t}
$$


assuming that $\phi_{0}, C_{e}, \alpha$, and $K_{u}$ are time-invariant. Substituting this relationship into equation (3), and accounting for the time derivatives (7) and (8) results in

$$
\sigma_{f} C_{e} \frac{\partial p}{\partial t}-\nabla \cdot[\hat{K} \nabla p]+R_{e} p+\hat{q}=0
$$

where $\hat{q}$ is given by

$$
\begin{gathered}
\hat{q}=q+\frac{\sigma_{f} \alpha}{K_{u}} \frac{\partial p_{o}}{\partial t}+\left(\phi_{0}+\frac{\alpha}{K_{u}} p_{o}\right) \frac{\partial\left(\Delta \rho S_{n}\right)}{\partial t}, \\
R_{e}=C_{e} \frac{\partial\left(\Delta \rho S_{n}\right)}{\partial t}
\end{gathered}
$$

and $\hat{K}$ is the effective permeability

$$
\hat{K}=\kappa K
$$

In the presence of two-phase effects the coefficients $\sigma_{f}$ and $\kappa$ will change with time. However, as shown next, in our application we shall be interested in the arrival time of a pressure disturbance as it propagates from a well. This transient pressure variation will generally propagate significantly faster than the two-phase saturation front. For this reason, we neglect the two-phase changes in $\sigma_{f}$ and $\kappa$ and treat them as constants. Then we can apply the Fourier transform to equation (12), resulting in the frequency domain equivalent,

$$
\nabla^{2} P+\Lambda \cdot \nabla P-i \omega D P=\hat{Q}
$$

where $P(\mathbf{x}, \omega)$ is the Fourier transform of the pressure, $\boldsymbol{\Lambda}$ is the gradient of the logarithm of conductivity,

$$
\mathbf{\Lambda}(\mathbf{x})=\nabla \ln \hat{K}(\mathbf{x})
$$

which vanishes for constant $\hat{K}(\mathbf{x})$,

$$
D(\mathbf{x})=\frac{\sigma_{f} C_{e}(\mathbf{x})}{\hat{K}(\mathbf{x})}
$$

is the reciprocal of the diffusivity, and $\hat{Q}$ is the Fourier transform of the normalized source term. If we include two-phase effects, equation (16) will contain convolutions because the coefficients $\sigma_{f}, \hat{K}$, and $R$ in equation (12) are now time-dependent. 


\section{An Asymptotic Solution to the Diffusion Equation}

It is not possible to derive an analytical solution of equation (16) for a general variation in $\hat{K}(\mathbf{x})$ and $C_{e}(\mathbf{x})$. Currently, numerical approaches are the most common methods for approximating solutions in arbitrary Earth models. Such numerical approaches are general but do not provide much insight into the nature of pressure propagation within a heterogeneous porous medium. Furthermore, as shown below, a semi-analytic solution has some advantages in treating the inverse problem, a central concern of this work. Thus, in this sub-section we highlight a semi-analytic solution to the diffusion equation, following upon the earlier work of Cohen and Lewis (1967), Virieux et al. (1994), Vasco et al. (2000b), and Shapiro et al. (2002). The approach, which is implemented in the frequency domain, is based upon the notion of a high frequency asymptotic solution, similar to methods used in the study of electromagnetic or elastic wave propagation (Friedlander and Keller 1955, Luneburg 1966, Kline and Kay 1979, Aki and Richards 1980, Kravtsov and Orlov 1990, Keller and Lewis 1995). However, the definition of 'high-frequency' is relative to the spatial and temporal variations in the background reservoir pressure in its undisturbed state, before injection or production. That is, though the transient pressure disturbance may take days or months to propagate away from a producing well, we can consider it as a high-frequency variation when compared to the background reservoir pressure which can vary over years, decades, or even longer. Naturally, if there are competing variations over the time scale in question, such as interference with nearby wells, we must account for that as a secondary source.

As noted by Virieux et al. (1994), an asymptotic solution to equation (16) is a power series in $1 / \sqrt{\omega}$,

$$
P(\mathbf{x}, \omega)=e^{-\sqrt{-i \omega} \sigma(\mathbf{x})} \sum_{n=0}^{\infty} \frac{A_{n}(\mathbf{x})}{(\sqrt{-i \omega})^{n}} .
$$

The form of the series (19) may be deduced on physical grounds, by considering a large argument expansion of the modified Bessel function of zeroth order, the solution 
to the diffusion equation for a homogeneous medium, (Virieux et al. 1994). The motivation for a series in powers of $1 / \sqrt{\omega}$ is similar to that for asymptotic methods used in modeling hyperbolic wave propagation. In particular, $\omega$ is large, the first term in the series (19)

$$
P(\mathbf{x}, \omega)=A_{0}(\mathbf{x}) e^{-\sqrt{-i \omega} \sigma(\mathbf{x})}
$$

or its time-domain equivalent obtained by inverse Fourier transforming equation (20),

$$
p(\mathbf{x}, t)=A_{0}(\mathbf{x}) \frac{\sigma(\mathbf{x})}{2 \sqrt{\pi t^{3}}} e^{-\sigma^{2}(\mathbf{x}) / 4 t}
$$

(Virieux et al. 1994, Vasco et al. 2000b), will accurately represent the solution to the diffusion equation. The expression (21) is similar in form to the solution of the diffusion equation in a homogeneous medium (Carslaw and Jaeger 1959) but with spatially varying coefficients $A_{0}(\mathbf{x})$ and $\sigma(\mathbf{x})$.

In order to obtain explicit expressions for $\sigma(\mathbf{x})$ and $A_{n}(\mathbf{x})$, which are required to fully specify the solution, the sum (19) is substituted into equation (16). The operators in equation (16) may be applied term by term to the series. The substitution of the expansion (19) into equation (16) produces an expression containing an infinite number of terms. Each term will contain $1 / \sqrt{\omega}$ to some power and we may consider sets of terms for any given order. As shown in Virieux et al. (1994) and Vasco et al. (2000b), the functions $\sigma(\mathbf{x})$ and $A_{0}(\mathbf{x})$ are determined by the terms of order $(1 / \sqrt{\omega})^{-2}$ and $(1 / \sqrt{\omega})^{-1}$ respectively. Because we are only interested in the phase, $\sigma(\mathbf{x})$, we only consider terms of order $(1 / \sqrt{\omega})^{-2} \sim \omega$ in the sub-section that follows.

We note that the expressions (20) and (21) are point-source solutions for a source which acts at a single location in space and at a single time. In applications, such as our synthetic and field examples discussed below, we shall use a step-function source which is a more realistic representation of fluid flow rates. Such sources, can be obtained by convolution with the point-source solution. In fact, any physical source can be modeled by convolution with a known source-time function. Alternatively, one 
could deconvolve the know source-time function from the observations and construct the equivalent point-source response. In our applications below, in which the source is approximated by a step-function, we simply work with the time-derivative of the pressure. By linearity, this is equivalent to the pressure values due to a delta-function source. There is an additional issue related to presence of the overburden pressure term in the source function $\hat{q}(\mathbf{x}, t)$ [equation (13)]. This term is not localized in space and may act over an interval of time. In the applications below we assume that the overburden stress does not change significantly with time and thus the derivative in equation (13) vanishes. However, as shown in Vasco (2004b), it is possible to correct for the overburden pressure changes, allowing for the use of the point-source expressions (20) and (21). The corrections provide a quantitative measure of the importance of variations in overburden stress in the changes of reservoir fluid pressure.

\section{The Eikonal Equation, Trajectories, and the Arrival Time of the Maximum Pressure Change}

If we consider terms of order $(\sqrt{-i \omega})^{2} \sim \omega$, we arrive at a scalar, nonlinear, partial differential equation for the phase $\sigma(\mathbf{x})$

$$
\nabla \sigma(\mathbf{x}) \cdot \nabla \sigma(\mathbf{x})-D(\mathbf{x})=0
$$

(Virieux et al. 1994, Vasco et al. 2000b). Equation (22), known as the eikonal equation, governs many types of propagation processes (Kline and Kay 1979, Kravtsov and Orlov 1990) and there are efficient numerical methods for its solutions (Sethian 1996). The eikonal equation relates the phase function $\sigma(\mathbf{x})$ to the flow properties $C_{e}(\mathbf{x})$ and $K(\mathbf{x})$, as contained in $D(\mathbf{x})$ [equation (18)]. We may also solve equation (22) using the method of characteristics (Courant and Hilbert 1962). In the method of characteristics, solutions are developed along particular trajectories, which are

denoted by $\mathbf{X}(l)$, where $l$ signifies position along the curve. The equations for the 
characteristic curves are a set of ordinary differential equations which depend upon $\sqrt{D}$

$$
\begin{gathered}
\frac{d \mathbf{X}}{d l}=\mathbf{s} \\
\frac{d \sigma}{d l}=\sqrt{D}
\end{gathered}
$$

where $\mathbf{s}=\nabla \sigma$ (Courant and Hilbert 1962). From equation (24), we can write the phase function as an integral

$$
\sigma(\mathbf{x})=-\int_{\mathbf{X}(l)} \sqrt{D} d l
$$

where $\mathbf{X}(l))$ is the trajectory from the injection well to the observation point $\mathbf{x}$.

The trajectory is found by solving the ray-equations (23) using a numerical technique. The method for determining the trajectory $\mathbf{X}(l)$ from a source point to a given observation point is known as the shooting method for a two-point boundary value problem (Press et al. 1992). Alternatively, we can determine the phase, and the trajectory, by simply post-processing the output of a numerical reservoir simulator. This approach, which eliminates the need for ray-tracing, is discussed in more detail in Vasco and Finsterle (2004) and Vasco (2004b). It makes use of the fact that

$$
\sigma=\sqrt{6 T_{\max }}
$$

where $T_{\max }$ is the time at which the pressure is a maximum. The relationship (26) is obtained by differentiating (21) with respect to time and setting the resulting expression to zero. One can post-process the results of a reservoir simulation to extract the arrival time of the peak of the pressure curve and hence $\sigma(\mathbf{x})$, using equation (26). The trajectories are found by integrating the differential equation (23) using a second-order Runge-Kutta technique (Press et al. 1992). This is a much simpler procedure than ray-tracing and essentially involves marching up the gradient of the phase field obtained from the simulator output (Vasco and Finsterle 2004). 


\section{A Linear Inversion Algorithm for Flow Properties}

Our ultimate goal is to estimate flow properties, based upon a set of measurements of deformation of the overburden. As discussed in the Appendix, we use the deformation data to estimate pressure variation within the reservoir induced by fluid injection or withdrawal. This procedure is illustrated by the synthetic and field examples below. For now, assume that we have estimates of pressure changes within the reservoir for a series of time intervals. That is, we have snapshots of the pressure changes for a sequence of times. If the time sampling is dense enough we can estimate the time at which the pressure change reaches a peak for points throughout the reservoir. Thus, using equation (26), we can estimate $\sigma(\mathbf{x})$, for $\mathbf{x}$ within a region

of the reservoir. As an example, let us denote the phase estimate at point $\mathbf{x}_{i}$ by the quantity $\Sigma_{i}$ and the integral by

$$
\Sigma_{i}=-\int_{\mathbf{X}_{i}} \sqrt{D} d l
$$

where $\mathbf{X}_{i}$ denotes the trajectory associated with the $\Sigma_{i}$ observation point. Given estimates of $\Sigma_{i}$ at points throughout the reservoir we can use equation (27) to infer $D(\mathbf{x})$ and consequently the flow properties which define this quantity [see equation (18)].

For a dense sampling of points we can calculate the trajectories directly from the estimates of phase, using equation (23),

$$
\frac{d \mathbf{X}}{d l}=\nabla \Sigma
$$

This, in effect, linearizes the inverse problem for $\sqrt{D}$, as given by equation (27) because, in estimating $\mathbf{X}_{i}$ directly from the phase values, we have removed the dependence of the trajectory on the unknown distribution of $D$. Another way to see this is to return to the eikonal equation (22), and substitute in the estimate $\Sigma(\mathbf{x})$ for the phase field. The result 


$$
\nabla \Sigma(\mathbf{x}) \cdot \nabla \Sigma(\mathbf{x})=D(\mathbf{x})
$$

is a linear equation which can be solved directly for $D(\mathbf{x})$. The linearity follows from the fact that we can estimate the phase field directly and do not consider it to be an unknown quantity.

By sub-dividing the Earth model into a set of grid blocks we can discretize the integral (27). Given a set of phase estimates, derived from field data, a set of data constraint equations will result. The resulting equations for the flow properties may be written in the form

$$
\Sigma=\mathrm{Gy}
$$

where $\boldsymbol{\Sigma}$ is a vector of phase estimates obtained from the deformation data, $\mathbf{G}$ is a coefficient matrix which contains the trajectory lengths in each grid block of the reservoir model, and $\mathbf{y}$ is the vector of flow property model parameters. They will consist of estimates of $\sqrt{D}$ in each grid block. If it is possible to estimate $C_{e}$ for the region of interest one can then solve for the effective permeability in each cell of the reservoir model.

Because the phase estimates $\boldsymbol{\Sigma}$ and the matrix coefficients of $\mathbf{G}$ contain errors, we do not solve equation (30) directly. Rather, we use the method of least squares to estimate the model parameters, the components of the vector $\mathbf{y}$ (Lawson and Hanson 1974) by minimizing the sum of the squares of the residuals. Due to the fact that the equations may be inconsistent and under-determined we also incorporate regularization or penalty terms into the inversion (Parker 1994). Our penalty terms consist of a norm penalty, which biases our solution towards a given initial model $\mathbf{y}_{0}$, and a roughness penalty which favors models which are smoothly varying. The penalty terms are quadratic forms defined over the space of model parameters. Thus, we minimize the sum

$$
R=|\mathbf{\Sigma}-\mathbf{G y}|_{2}+W_{n}\left|\mathbf{y}-\mathbf{y}_{0}\right|_{2}+W_{r}|\mathbf{D y}|_{2}
$$


where $|\mathbf{z}|_{2}$ signifies the $L_{2}$ norm of the vector $\mathbf{z}, W_{n}$ and $W_{r}$ are scalar coefficients controlling the importance of the penalty terms in relation to the importance of fitting the data, and $\mathbf{D}$ is a matrix which approximates a differencing operator. Because the quantity $R$ is a quadratic function of the model parameters, the condition for it to be a minimum is a linear system of equations. We solve this system of equations using the iterative solver first proposed by Paige and Saunders (1982).

\section{APPLICATIONS}

\section{Numerical Illustration}

As noted above, there are several steps in the estimation procedure, each involving some degree of approximation. For example, the phase field, $\Sigma(\mathbf{x})$, is inferred from the pressure estimates, which are in turn derived from the deformation data. In order to illustrate the steps in our approach, and to examine how well the technique works under favorable conditions, we consider a set of synthetic deformation values. The test case was designed to roughly correspond to the actual field case that we discuss below. A reference permeability model, shown in Figure 1, was used to simulate the pressure response due to the initiation of fluid injection. As in the field case, the injection started suddenly and continued for over two years. For this test we modeled the flow rate using a step-function. The reservoir model consisted of a 20 meter thick layer, lying at a depth of 2,000 meters. The numerical reservoir simulator TOUGH2 (Pruess et al. 1999) was used to compute the evolution of pressure within the reservoir due to the fluid injection. The code solves the pressure equation (12) numerically, using an integral finite differences technique (Pruess et al. 1999).

The pressure variations within the reservoir are used to compute reservoir volume change assuming a linear poroelastic constitutive model (Rice and Cleary 1976, Segall 1985). That is, the volume changes $\mathbf{v}_{f}$ in the reservoir are given by 


$$
\mathbf{v}_{f}=\Pi^{-1} \delta \mathbf{p}
$$

where $\delta \mathbf{p}$ are the pressure changes, and $\boldsymbol{\Pi}^{-1}$ is the inverse of the coefficient matrix $\Pi$ which is given in the Appendix [equation (A3)]. In calculating the volume change within the reservoir we can account for stress transmission within the overburden. This is done for a homogeneous poroelastic overburden using the method described in Segall (1985). More complicated models of the overburden can be treated using more sophisticated numerical codes (Vasco et al. 2000a). However, factors such a layering do not significantly influence the modeling of deformation due to volume change at depth (Battaglia and Segall 2004).

The volume change within the reservoir was used to compute the deformation within the homogeneous overburden using a generalization of Maruyama's (1964) method (Vasco et al. 1988, Vasco et al. 2000a). In particular, we computed the range change, the change in distance to a hypothetical point in space, as discussed in Vasco et al. (2002) and Vasco and Ferretti (2005). Range change is measured by analyzing radar reflection data from orbiting satellites and provides a very accurate measure of surface displacement at high spatial densities and over large regions of the Earth (Massonnet and Feigl 1998, Burgmann et al. 2000, Ferretti et al. 2000, Ferretti et al. 2001, Colesanti et al. 2003). For the numerical illustration we calculated range change on a 40 by 40 grid of values at the surface, for 14 irregularly spaced time intervals $[24,59,94,129,164,199,269,304,409,549,584,689,724,864$ days]. The time intervals correspond to those in the actual set of range change observations described below. The range change values for four of the intervals are shown in Figure 2. Note the smoothly varying changes, with a peak range change of approximately $1.0 \mathrm{~cm}$.

The first step in the inversion procedure involves estimating the volume change within the reservoir from the range change observations. This step has been discussed extensively in other papers (Vasco et al. 1988, Vasco et al. 1998, Mossop and Segall 
1999, Vasco et al. 2000a, Du and Olson 2001, Vasco et al. 2001, Vasco et al. 2002, Vasco and Ferretti 2005) and is outlined in the Appendix. It requires the solution of a linear inverse problem [equation (A1)], in which the unknown parameters are grid block fractional volume changes and the observations are range change data. A

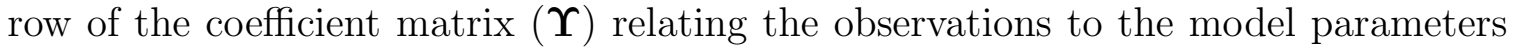
consists of the response of the overburden to a fractional change in the volume of a grid block. The grid blocks are identical to those used in the forward calculation, with a thickness of $20 \mathrm{~m}$. The entries of $\Upsilon$ depend on the spatial distribution of the elastic properties of the overburden. For this illustration we assume a homogeneous elastic half-space which is characterized by its Poisson's ratio [assumed to be 0.25]. Details of the computation are discussed in Vasco et al. $(1988,2002)$ and Vasco and Ferretti (2005).

Next the estimated volume changes are mapped into reservoir pressure changes. This requires estimates of the mechanical properties of the reservoir rock. In particular, we require the bulk modulus, as contained in $\Pi^{-1}$ in equation (32), throughout the reservoir. We should note that, for the arrival time approach taken here, we do not have to take this step. Rather, we can work directly with the fractional volume change in estimating the arrival time. As long as there is a linear relationship between the volume change and the pressure change, and the relationship does not contain time derivatives, as in a viscous medium, the arrival time estimates will be identical. This is one advantage of working with a kinematic, or travel time approach, rather than working with amplitudes themselves. The estimated pressure changes for four time intervals $[24,128,408$, and 842 days] are shown in Figure 3. The peak pressure change is centered on the injection well and decays rapidly with distance. The pressure change is somewhat asymmetric, reflecting the heterogeneity within the reservoir. In order to emphasize the propagation of the transient pressure change we estimated the time derivative of the pressure from the sequence of 14 time intervals. The resulting time derivatives, normalized by the peak values in each grid block, are 
shown in Figure 4. One can clearly observe the propagation of the peak pressure derivative away from the injection well as a function of time.

From the pressure derivatives we estimate the arrival time of the peak over the simulation grid. That is, using the pressure time series for each grid block we estimate the time derivative and the time at which the derivative reaches a peak value. The resulting distribution of sampled arrival times are shown in Figure 5. The distribution of travel times is not symmetric about the injection well, an indication of the heterogeneous propagation of the pressure disturbance. The pressure arrival times, shown in Figure 5, form the basic data for an inversion for flow properties. They are used to estimate the phase field and thus compute the elements of the vector $\Sigma$ in equation (30). Furthermore, as noted above, the phase field estimates may be used to define the trajectories $\mathbf{X}_{i}$ in the integral (27) or its discrete equivalent. The trajectories are used to calculate the matrix coefficients of $\mathbf{G}$ in equation (30), resulting in a linear inverse problem. The trajectories, or raypaths, computed from the phase field are also shown in Figure 5. The regularized linear inverse problem, entails minimizing the quadratic form $R$, as defined in the expression (31). The linear system of equations, comprising the necessary equations for a minimum of $R$, are solved using the iterative linear solver LSQR (Paige and Saunders 1982).

The effective permeability estimates produced by the inversion algorithm are shown in Figure 6. The model contains the large-scale features which are present in the reference model. In particular, there are high permeabilities to the north and west of the injection well and low permeabilities to the southeast. The overall pattern is a smoothed version of the reference model (Figure 1), reflecting the influence of the roughness and norm penalties. Due to the limited extend of the phase data, it is not possible to estimate flow properties outside of a region surrounding the injection well. Stated another way, it is not possible to infer flow properties beyond the region over which the pressure changes have propagated during the observation interval [0-864 days]. Another limiting factor is the decay of pressure with distance: after 
the disturbance has propagated a certain distance from the well the changes will be small, producing no observable deformation at the surface. The sampling shown in Figure 5, identifies the region over which estimates are reliable. This region roughly corresponds to the area of significant permeability deviations from the background model (Figure 6).

The regularization penalty terms in $R$ [equation (31)] will trade-off with the fit to the arrival times. By increasing the regularization weights $W_{n}$ and $W_{r}$ we can degrade the arrival time match. Thus, after completing the inversion it is important to examine the fit to the arrival times to ensure that they were not over- or underfit. The match is shown in Figure 7, in terms of deviations from a reference arrival time, calculated using a homogeneous starting model. In general, the calculated deviations from the reference predictions appear to match the reference deviations rather well. The inverse problem is linear and one can compute the model parameter resolution matrix as part of a formal model assessment (Aki and Richards 1980, Parker 1994). Because the focus of the paper is to introduce an approach for estimating flow properties from deformation data, we shall not compute model parameter resolution estimates.

\section{Application to Permanent Scatterer Observations from the Krechba Field, Al- geria}

In this section we describe an application of the methodology to range change data associated with $\mathrm{CO}_{2}$ injection in the Krechba field, Algeria. The Krechba, Teg, and Reg are gas fields are distributed along a north-northwest trending anticline. The producing layer $\mathrm{C} 10.2$, is a roughly twenty meter thick layer of quartzoze, fine grained sandstone of Lower Carboniferous age. The production zone is overlain by at least one kilometer of dark gray to black, subfissile mudstones. Above the mudstone there is another kilometer of interbedded mudstones and sandstones. The gas produced 
from these fields contains a high mole fraction of $\mathrm{CO}_{2}$ which must be removed and disposed of for economic and environmental reasons. The $\mathrm{CO}_{2}$ gas is separated from the hydrocarbons and is re-injected into the reservoir at three nearby wells, KB-501, KB-502, and KB-503. The $\mathrm{CO}_{2}$ injection wells are in the same formation as the producers. The horizontal wells are located on the flanks of the anticline, at depths of roughly two kilometers. The well KB-501, which we consider in this study, lies roughly $8 \mathrm{~km}$ to the east-northeast of the production wells, about $100 \mathrm{~m}$ lower in elevation. Mud losses during the drilling of numerous wells indicate flow into preexisting fractures, dipping at approximately $81^{\circ}$, striking $\mathrm{N} 43^{\circ} \mathrm{W}$. The direction is sub-parallel to the regional stress direction determined for the Reg and Teg fields and observed throughout Algeria. The stress directions in the gas fields are remarkably consistent, striking $315^{\circ}$, a sign that the horizontal stress field is highly anisotropic. Breaks in the topography of the structure map of the top of the reservoir layer, as determined by seismic imaging, indicate possible faults on the flanks of the anticline, trending north-northwest.

As part of a $\mathrm{CO}_{2}$ sequestration research and development program, Berkeley Laboratory and Tele-Rilevamento Europa (TRE) explored the use of Interferometric Synthetic Aperture (InSAR) data for monitoring $\mathrm{CO}_{2}$ injections. One goal of this work was to identify flow paths at depth and geologic features controlling flow. The injection of $\mathrm{CO}_{2}$ into the water column induces multiphase flow because the reservoir is initially water-filled. At reservoir pressure the $\mathrm{CO}_{2}$ behaves as a super-critical fluid, with a viscosity and density somewhat different from water. The well head injection pressure was variable, on average a pressure of $15 \mathrm{MPa}$ was maintained after the starting of pumping, with peaks approaching $18 \mathrm{MPa}$ early in the injection and about seven months into the injection. At these pressures super-critical $\mathrm{CO}_{2}$ should have a density greater than 0.85 grams $/ \mathrm{cm}^{3}$. In the region of interest, the reservoir does not vary significantly in depth, and the density differences do not impact the flow. Furthermore, the advective flow almost certainly dominates the geochemical changes 
at depth for the two to three years of injection that we considered. We model the $\mathrm{CO}_{2}$-water system as an equivalent single phase for the pressure calculations which should be acceptable given the reservoir pressure conditions and the time interval (Kumar et al. 2005).

This study utilized satellite radar images of the European Space Agency (ESA) Envisat archive from July 12, 2003 through March 19, 2007. Two satellite tracks, number 65 and number 294, surveyed the region during the $\mathrm{CO}_{2}$ injection, containing 26 and 18 images, respectively. The data were processed using a permanent scatterer technique developed by Politenico di Milano and TRE (Ferretti et al. 2000, 2001, Colesanti et al. 2003). In this approach, stable scatterers are identified using a statistically-based analysis of the phase and amplitude characteristics of the energy scattered from the Earth's surface. A subset of scatterers is identified as Permanent Scatterer Candidates and used to estimate atmospheric and orbital errors. The atmospheric and orbital biases in the signal phase are corrected for, and estimates of range change are derived from the phase shifts between pairs of back-scattered radar signals. The range change represents the change in distance from a point on the Earth's surface and the nominal satellite orbit. Thus, range change is the change in the length of a hypothetical line connecting the observation location and the sensor location. Estimates of range change are influenced by variations in atmospheric moisture in space and time, corrections for topography, and land surface changes such as growing vegetation.

Given a sequence of images, one can estimate the average range change velocity over a given time interval. That is, the average change in distance to the satellite orbit over the given time interval. Similar to Differential Global Positioning Satellite (DGPS) data, all InSAR observations are actually differential measurements with respect to a reference point which is assumed to be motionless. For example, in Figure 8 we plot the average range velocity for track 65 and track 294, corresponding to the region around the $\mathrm{CO}_{2}$ injector KB-501. In this image, one can see the range 
change due to uplift generated by the $\mathrm{CO}_{2}$ injection at the well. The quality of the data for this region is high due to the surface characteristics of the region, the area is primarily rock outcrops and desert with little shifting sand. The peak range velocity in Figure 8 is greater than $5 \mathrm{~mm} /$ year, which is several times larger than the estimated standard deviation of $1 \mathrm{~mm} /$ year.

The noise in the estimates is discernible if we compare the range velocity inferred for Tracks 65 and 294. Each image contains notable scatter and there are differences between the two range velocity estimates. It should be noted that the time span covered by the two data sets is not identical and that the number of data available is less then is typical used in permanent scatterer InSAR (Ferretti et al. 2000, 2001, Colesanti et al. 2003). This may lead to differing atmospheric corrections resulting in a long period spatial variation between the two tracks. Furthermore, the angular view of the Earth's surface is slightly different for the two data sets. The well location is situated differently in each track. The location of KB-501 is much closer to the edge of the image in Track 65 .

In an effort to improve the signal-to-noise ratio, we stacked the range change estimates for Tracks 65 and 294, interpolating them onto a common time sampling. The time sampling was identical to that in our numerical study, consisting of 14 time intervals $[24,59,94,129,164,199,269,304,409,549,584,689,724,864$ days]. Furthermore, we averaged the scatterers spatially, using a moving window with a radius of 10 points. The resulting stacked and averaged estimates are shown in Figure 9 for four time intervals [24, 128, 408, and 842 days]. The range change clearly increases with time, to a peak value of over $1 \mathrm{~cm}$, and appears to migrate to the northwest.

The range change data were then inverted for volume change within the reservoir, using the approach described in Vasco et al. (2002) and Vasco and Ferretti (2005), and outlined in the Appendix. In essence, the linear system (A1) is solved for the volume changes in the reservoir. The Green's function $g_{i}\left(\mathbf{x}_{k}, \mathbf{y}\right)$ used in the inversion 
[see equation (A2)] corresponded to a homogeneous half-space. The overburden is composed of layers of mudstone and quartzoze sandstone. Such layering does not significantly influence the estimates of volume change (Battaglia and Segall 2004), and a half-space model is thought to be an acceptable approximation. Because the reservoir itself was two kilometers deep and only varied in depth by a few tens of meters in the region of interest, we approximated it by a horizontal layer. Because we did not have any direct pressure measurements, there were only deformation data, $\mathbf{u}(t)$ in the inversion. The inversion was regularized by including roughness and model norm penalty terms. Furthermore, volume changes were penalized based upon their distance from the injection well KB-501. That is, volume changes near well KB-501 were favored over more distant changes. Thus, only the well location contributed to the inversion result, no information about flow rates within the well was available.

The volume change was then mapped into reservoir pressure change, based upon equation (A5). Laboratory tests indicated that a value of $K_{u}$ of $10.0 \mathrm{GPa}$ was representative of the mechanical behavior of the overburden. The inferred reservoir pressure changes for time intervals of 24, 128, 408, and 842 days are shown in Figure 10. The peak pressure change of approximately $20 \mathrm{MPa}$, is compatible with measurements of reservoir pressure from a downhole sensor. The migration of pressure change to the northwest is evident from the sequence of snapshots.

From the sequence of pressure changes we estimate the phase or travel time of the disturbance due to the onset of $\mathrm{CO}_{2}$ injection. Because the flow rate was non-uniform, there were several peaks in the time series of the pressure derivative. We focused on the time associated with the first peak of pressure derivative in each grid block. A more sophisticated approach, which we may attempt in a future study, involves deconvolving the flow rate function from the time series of each grid block to produce the response to a step function in flow rate. In Figure 11, we plot the normalized time derivative associated with the first peak after 305 days of pumping. From this figure it is evident that the pressure variation has propagated more rapidly to the 
northwest. In Figure 12a the square root of the estimated arrival time is plotted for the region surrounding the injection well KB-501.

The arrival time values form the basic data set for the inversion for flow properties. The estimates were constructed for a 40 by 40 grid of cells defining the reservoir model. However, it is only possible to estimate arrival times for those grid blocks to which the pressure change had propagated during the observation interval. The active grid blocks are shown in Figure 12b, where the block size is proportional to the square root of the arrival time. As noted in the Methodology section, the phase field defines the trajectories $\mathbf{X}(l)$, as evident in equation (28). The trajectories associated with our phase estimates are also shown in Figure 12b, as curves connecting the phase points to the injection well. The trajectories are used to construct the coefficients of the matrix $\mathbf{G}$, which follow from a discretization of the integral (27) to a summation. The approach is identical to that used in travel time tomography (Aki and Richards 1980). However, the inverse problem is linear because we have used the phase field estimates to calculate the trajectories directly.

A regularized inversion of the linear system, equation (30), equivalent to the necessary equations for a minimum of $R$ in equation (31), was used to estimate the permeability variations in the region surrounding the pumping well (Figure 13). The coefficients $W_{n}$ and $W_{r}$ were found by trial and error, the result of a number of trial inversions. The goal was to find a smooth model which fit the estimated arrival times within the expected error. The permeability multipliers produced by the inversion algorithm suggest a linear, high-permeability zone cutting across the injection well and trending northwest. This feature is compatible with the migration of deformation to the northwest, which is notable in Figure 9. The orientation of the high permeability zone is parallel to the trend of the structural anticline which defines the field. Furthermore, the location of the high permeability feature correlates with a break in topography which suggests a fault cutting the eastern side of the anticline. The inferred fault is indicated by the dashed line in Figure 13. The fault may ex- 
tend southward, it is difficult to constrain the trace from the seismic structure map. Thus, it seems that the flow is controlled by a fault and the permeability model is in accordance with the geologic structure of the reservoir.

Next, we examine the fit to the arrival time data provided by the permeability model (Figure 14). Specifically, Figure 14 displays the fit to the phase data which were derived from the deformation [range change] measurements. Thus, the 'data' are actually estimates produce by first inverting the range change for pressure change and then estimating the arrival time from the time-varying pressures. Each inversion will introduce some level of approximation and bias due to the inclusion of regularization terms and also due to errors in the actual measurements. Overall, the travel times predicted by the permeability model appear to agree with the observed travel times.

Finally, we consider the influence that variations in the range change data have on our permeability estimates. In particular, there are differences in the range change velocity estimates for tracks 65 and 294, which are visible in Figure 8. In order to examine the stability of our permeability estimates we conducted an inversion in which only data from track 65 was used. The resulting phase estimates are shown in the upper panel of Figure 15. The general features of these estimates: the asymmetric distribution of the phase, the faster propagation to the northwest, and the overall extent of the contours, agree with the averaged range change estimates (Figure 12a). Furthermore, the phase estimates from track 65 are in qualitative agreement with estimates from track 294 (Figure 15, lower panel). However, there are some difference in detail between the various estimates. An inversion of the phase data from tracks 65 and 294, results in the permeability multipliers shown in Figure 16. Again, the general features of the permeability estimates, such as the northwest trending linear high permeability anomaly and the magnitude of the deviations, are the same for the two inversions. The primary differences in the results are the southeast extent of the high permeability trend. There is also a slight shift of the anomalies to the southwest in the inversion of data from track 294, relative to the inversion results from track 
65. This shift may be due to the fact that we used the same look vector for the two inversions. In reality the satellites were in somewhat different positions for the two tracks.

\section{DISCUSSION AND CONCLUSIONS}

Fluid pressure changes within a reservoir or aquifer can produce measurable deformation within the overburden. Such deformation has been observed in both geodetic data (Castle et al. 1969, Evans et al. 1982, Dussealt et al. 1993, Bruno and Bilak 1994, Chilingarian et al. 1994, Castillo et al. 1997, Fielding et al. 1998, Wright 1998, Mossop and Segall 1999, Du and Olson 2001, Stancliffe and van der Kooij 2001, Vasco et al. 2002, Du et al. 2005, Vasco and Ferretti 2005) and time-lapse seismic surveys (Guilbot and Smith 2002, Hatchell and Burne 2005, Hawkins et al. 2007, Rickett et al. 2007, Staples et al. 2007). Furthermore, current and planned instrumentation, such as permanent sea floor sensors and high precision pressure gauges (Sasagawa et al. 2003) can generate useful displacement measurements. The resulting deformation can be used to infer volume and pressure changes within the reservoir (Vasco et al. 1998, Mossop and Segall 1999, Vasco et al. 2000a, Du and Olson 2001, Vasco et al. 2001, Du et al. 2005, Vasco and Ferretti 2005, Hodgson et al. 2007).

While it is possible to map the estimated pressure changes directly into flow properties (Vasco et al. 2001, Vasco and Ferretti 2005), in this paper we use an alternative approach, based upon the idea of a pressure 'arrival time' (Vasco et al. 2000b, Vasco 2004b). The primary advantage of this approach is that it is not sensitive to the mechanical properties within the reservoir. That is, if the reservoir behaves elastically over each time increment, the arrival time estimate can be derived from the volume change within the reservoir and does not require mapping the volume change into pressure change. Thus, we do not need to estimate the value of $K_{u}$, and its variation, within the reservoir [see equation (A6)]. An additional advantage 
of this approach is that the phase field is estimated from the deformation data and can be used to compute the raypaths or trajectories for the tomographic travel time inversion. This, in effect, linearizes the inverse problems and one then has all the tools of linear inverse theory available (Parker 1994). Thus, the estimation of flow properties, based upon deformation data, is a linear problem.

Our application of the technique to InSAR range change estimates from the Krechba field in Algeria indicates that the approach works in practice. The linear, high-permeability feature produced by the inversion is in accordance with the local and regional geology. Specifically, the feature lies at the southern extension of a northwest trending fault on the flank of the structural anticline defining the field. This suggests that a fault is controlling the flow of $\mathrm{CO}_{2}$ from the injection well. There is the possibility that the fault is deforming in response to the injected fluid. This mechanism might involve changes in permeability due to deformation, a subject not addressed in this work.

One of the limitations of this approach is the need for adequate sampling of the deformation field in time and space. In the near future a growing number of satellite platforms, mounting high resolution radar sensors will become available [eg. Radarsat-2, Terrasar-X, Cosmo-Skymed] providing additional coverage. Dense spatial sampling in three-dimensions can also be obtained through the use of time-lapse seismic data, though at a higher cost. Permanent sea floor sensors could be used to improve the sampling in time. Also, it is possible to apply the arrival time methodology to pressure estimates obtained directly from time-lapse seismic data (Tura and Lumley 1999, Landro 2001), by-passing the estimation of deformation entirely. In addition, developments such as side-scan-sonar interferometry (Chang et al. 2000) and acoustic positioning (Spiess et al. 1998), may provide useful data. An extension of this work would allow for permeability changes to accompany deformation. That is, there may be coupling between deformation and flow properties. Such coupling might occur when the $\mathrm{CO}_{2}$ is channeled into a narrow fault zone (Murphy et al. 2004). 
We plan on considering pressure-dependent flow properties, and their estimation, in a future study.

\section{ACKNOWLEDGMENTS}

This work was supported by the Assistant Secretary, Office of Basic Energy Sciences and the GEOSEQ project for the Assistant Secretary for Fossil Energy, Office of Coal and Power Systems through the National Energy Technology Laboratory of the U. S. Department of Energy under contract DE-AC02-05CH11231. The permanent scatterer (PS) data were processed by Tele-Rilevamento Europa (TRE), a spin-off company of Politecnico di Milano, world-wide exclusive licensee of the Polimi PS Technique $^{\mathrm{TM}}$. The authors wish to thank ESA for all satellite data used in this study and the entire TRE staff for supporting the SAR data processing. The In Salah $\mathrm{CO}_{2}$ Joint Industry Project (BP, StatoilHydro, and Sonatrach) is thanked for provision of production, injection, and subsurface data. Additional computations were carried out at the Center for Computational Seismology, Berkeley Laboratory. 


\section{REFERENCES}

Aki, K., and Richards, P. G., 1980, Quantitative seismology: Freeman and Sons.

Amelung, F., Galloway, D. L., Bell, J. W., Zebker, H. A., and Laczniak, R. J., 1999, Sensing the ups and downs of Las Vegas-InSAR reveals structural control of land subsidence and aquifer-system deformation: Geology, 27 , 483-486.

Arts, R., Eiken, O., Chadwick, A., Zweigel, P., van der Meer, L., and Zinsznet, B., Monitoring $\mathrm{CO}_{2}$ injected at Sleipner using time-lapse seismic data: Energy, 29, 1383-1392.

Audet, D. M., and Fowler, A. C., 1992, A mathematical model for compaction in sedimentary basins: Geophys. J. Int, 110, 577-590.

Barker, R., and Moore, J., 1998, The application of time-lapse electrical tomography in groundwater studies: The Leading Edge, 17, 1454-1458.

Barkved, O. I., and Kristiansen, T., 2005, Seismic time-lapse effects and stress changes: Examples from a compacting reservoir: The Leading Edge, 24, 12441248.

Battaglia, M., and Segall, P., 2004, The interpretation of gravity changes and crustal deformation in active volcanic areas, Pure and Applied Geophysics, 161, 1453-1467.

Barr, F., 1973, Feasibility study of seismic-reflection monitoring system for underground waste-material injection sites: AAPG Bull., 57, 1591-1592.

Behrens, R., Condon, P., Haworth, W., Bergeron M., Wang, Z., and Ecker, C., 2002, Monitoring of water influx at Bay Marchand: The practical use of 4D in an imperfect world: SPE Res. Eval. and Eng., SPE 79961 (October), 410-420. 
Binley, A., Winship, P., Middleton, R., Magdeline, P., and West, J., 2001. Highresolution characterization of vadose zone dynamics using cross-borehole radar, Water Resources Research, 37, 2639-2652.

Biot, M. A., 1941, General theory of three-dimensional consolidation: J. Appl. Phys, $12,155-164$.

Brewster, M. L., and Annan, A. P., 1994, Ground-penetrating radar monitoring of a controlled DNAPL release: 200 MHz radar: Geophysics, 59, 1211-1221.

Bruno, M. S., and Bilak, R. A., 1994, Cost-effective monitoring of injected steam migration using surface deformation analysis: SPE Western Regional Mtg., 27888 , 397-412.

Burkhart, T., Hoover, A. R., and Flemings, P. B., 2000, Time-lapse (4D) seismic monitoring of primary production of turbidite reservoirs as South Timbalier Block 295, offshore Louisiana, Gulf of Mexico: Geophysics, 65, 351-367.

Butler, J. J., C. D., McElwee, and G. C. Bohling, 1999, Pumping tests in networks of multilevel sampling wells: Motivation and methodology: Water Resour. Res., 35, 3553-3560.

Burgmann, R., R. A. Rosen, and E. J. Fielding, 2000. Synthetic aperture radar interferometry to measure Earth's surface topography and its deformation: Ann. Rev. Earth Planetary Sci., 28, 169-209.

Carslaw, H. S., and Jaeger, J. C., 1959, Conduction of Heat in Solids: Oxford University Press, Oxford.

Castillo, W., Hunter, S., Harben, P., Wright, C., Conant, R., and Davis, E., 1997, Deep hydraulic fracture imaging: Recent advances in tiltmeter technologies: Int. J. Rock. Mech. and Min, Sci., 34 , 3-4. 
Castle, R. O., Yerkes, R. F., and Riley, F. S., 1969, A linear relationship between liquid production and oil-related subsidence: in Tison, L. J., (Ed.), Land subsidence, 1 , Internat. Assoc. Sci. Hydrology Publ., (88), 597-604.

Chang, E., Marx, D. S., Nelson, M. A., Gillespie, W. D., Putney, A., Warman, L. K., Chatham, R. E., and Barrett, B. N., 2000, Long range active synthetic aperture sonar, results: Oceans 2000 Conference, Paper DTW-9901-00002, Providence R.I.

Chilingarian, G. V., Donaldson, E. C., and Yen, T. F., 1994, Subsidence due to fluid withdrawal: Elsevier Science.

Cohen, J. K., and R. M. Lewis, 1967, A ray method for the asymptotic solution of the diffusion equation, J. Inst. Maths. Applics., 3, 266-290.

Colesanti, C., Ferretti, A., Prati, C, and Rocca, F., 2003, Monitoring landslides and tectonic motions with the permanent scatterers technique: Engin. Geol., 68 , 3-14.

Cook, P. J., 1995, Analysis of Interwell Hydraulic Connectivity in Fractured Granite, M. S. thesis, University of California, Berkeley.

Courant, R., and Hilbert, D., 1962, Methods of Mathematical Physics, Interscience, New York.

de Marsily, G., 1986, Quantitative hydrogeology: Academic Press.

Du, J., and Olson, J. E., 2001, A poroelastic reservoir model for predicting subsidence and mapping subsurface pressure fronts: J. Petro. Sci. and Eng., 30 , 181-197.

Du, J., Brissenden, S. J., McGillivray, P., Bourne, S., Hofstra, P., Davis, E. J., Roadarmel, W., H., Wolhart, S. L., Marsic, S., Gusek, R., and Wright, C. A., 2005, Mapping reservoir volume changes during cyclic steam stimulation using tiltmeter based surface deformation measurements, 2005 SPE International Thermal Oper- 
ations and Heavy Oil Symposium, CHOA 97848, Calgary, Alberta, Canada, 1-3 November 2005.

Dussealt, M. B., Bilak, R. A., and Rothenburg, L., 1993, Inversion of surface displacements to monitor in-situ processes: Int. J. Rock Mech. and Min. Sci., 30 , 1219-1222.

Eastwood, J., Lebel, J. P., Dilay, A., and Blakeslee, S., 1994, Seismic monitoring of steam-based recovery of bitumen: The Leading Edge, 4, 242-251.

Evans, K. F., Holzhausen, G. R., and Wood, M. D., 1982, The geometry of a largescale nitrogen gas hydraulic fracture formed in Devonian shale: An example of fracture mapping using tiltmeters: SPE J., 22, 755-763.

Fielding, E. J., Blom, R. G., and Goldstein, R. M., 1998, Rapid subsidence over oil fields measured by SAR interferometry, Geophys. Research Letters, 25, 3215-3218.

Ferretti, A., Prati, C, and Rocca, F., 2000, Nonlinear subsidence rate estimation using permanent scatterers in differential SAR interferometry: IEEE Trans. Geo. and Remote Sensing, 38, 2202-2212.

Ferretti, A., Prati, C, and Rocca, F., 2001, Permanent scatterers in SAR interferometry: IEEE Trans. Geo. and Remote Sensing, 39 , 8-20.

Freyberg, D. L., 1986, A natural gradient experiment on solute transport in a sand aquifer, 2, Spatial moments and the advection and dispersion of nonreactive tracers, Water Resour. Res., 22, 2031-2046.

Friedlander, F. G. and Keller, J. B., 1955, Asymptotic expansions of solutions of $\left(\nabla^{2}+k^{2}\right) u=0$. Comm. Pure Appl. Mat., 8, 387.

Galloway, D. L., K. W. Hudnut, S. E. Ingebritsen, S. P. Phillips, G. Peltzer, F. Rogez, and P. A. Rosen, 1998, Detection of aquifer system compaction and land 
subsidence using interferometric synthetic aperture radar, Antelope Valley, Mojave Desert, California: Water Resour. Res., 34, 2573-2585.

Greaves, R. J., and Fulp, T., 1987, Three dimensional seismic monitoring of an enhanced oil recovery process: Geophysics, 52, 1175-1187.

Gringarten, A. C., T. von Schroeter, T. Rolfsvaag, and J. Bruner, 2003, Use of downhole permanent pressure gauge data to diagnose production problems in a North Sea horizontal well: Paper SPE 84470 presented at the SPE Annual Technical Conference and Exhibition, Denver, Colorado, October 5-October 8.

Guilbot, J. and Smith, B., 2002, 4-D constrained depth conversion for reservoir compaction estimation: Application to Ekofisk field: The Leading Edge, 21 , 302-308.

Hall, S. A., 2006, A methodology for 7D warping and deformation monitoring using time-lapse seismic data: Geophysics, 71, O21-O31.

Hatchell, P. and Bourne, S., 2005, Rocks under strain: Strain-induced time-lapse time shifts are observed for depleting reservoirs: The Leading Edge, 17, 1222-1225.

Hawkins, K., Howe, S., Hollingworth, S., Conroy, G., Ben-Brahim, L., Ttindle, C., Taylor, N., Joffroy, G., and Onaisi, A., 2007, Production-induced stresses from time-lapse time shifts: A geomechanics case study from Franklin and Elgin Fields: The Leading Edge, 26 , 655-662.

Hodgson, N., MacBeth, C., Duranti, L., Rickett, J., and Nihei, K., 2007, Inverting for reservoir pressure change using time-lapse time strain: Application to the Genesis Field, Gulf of Mexico, The Leading Edge, 26 , 649-652.

Hoffmann, J., H. A. Zebker, D. L. Galloway, and F. Amelung, 2001, Seasonal subsidence and rebound in Las Vegas Valley, Nevada, observed by synthetic aperture radar interferometry, Water Resour. Res., 37, 1551-1566. 
Hoversten, G. M., R. Gritto, J. Washbourne, and T. Daley, 2003, Pressure and fluid saturation prediction in a multicomponent reservoir, using combined seismic and electromagnetic imaging: Geophysics, 68, 1580-1591.

Hsieh, P. A., S. P. Neuman, G. K. Stiles, and E. S. Simpson, 1985, Field determination of the three-dimensional hydraulic conductivity tensor of anisotropic media, 2, Methodology and application to fractured rocks: Water Resour. Res., 21, 16671676.

Huang, X., Meister, L., and Workman, R., 1998, Improving production history matching using time-lapse seismic data: The Leading Edge, 17, 1430-1433.

Johnston, D. H., McKenny, R. S., Verbeek, J. and Almond, J., 1998, Time-lapse seismic analysis of Fulmar Field: The Leading Edge, 17, 1420-1428.

Karasaki, K., B. Freifeld, A. Cohen, K. Grossenbacher, P. Cook, and D. Vasco, 2000, A multidisciplinary fractured rock characterization study at the Raymond field site, Raymond California, J. Hydro., 236, 17-34.

Keller, J. B., and Lewis, R. M., 1995. Asymptotic methods for partial differential equations: The reduced wave equation and Maxwell's Equations, in Surveys in Applied Mathematics, 1, Keller, J. B., McLaughlin D. W, and Papanicoulaou (eds.), 1-82, Plenum Press, New York.

Kline, M., and Kay, I. W., 1979. Electromagnetic Theory and Geometrical Optics, Robert E. Krieger Publishing, Huntington, NY.

Kravtsov, Y. A., and Y. I. Orlov, 1990, Geometrical Optics of Inhomogeneous Media, Springer-Verlag, Berlin.

Kumar, A., Ozah, R. C., Noh, M., Pope, G., Sepehrnoori, K., Bryant, S., and Lake, L. W., 2005, Reservoir simulation of $\mathrm{CO}_{2}$ storage in deep saline aquifers, SPE J., 10, 336-343. 
Landro, M., 2001, Discrimination between pressure and fluid saturation changes from time-lapse seismic data: Geophysics, 66 , 836-844.

Landro, M., and Stammeijer, J., 2004, Quantitative estimation of compaction and velocity changes using 4D impedance and traveltime changes: Geophysics, 69 , 949-957.

Lawson, C. L., and Hanson, R., 1974, Solving Least Squares Problems: Prentice-Hall, Englewood Cliffs, NJ.

Lazaratos, S. K., and Marion, B. P., 1997, Crosswell seismic imaging of reservoir changes caused by $\mathrm{CO}_{2}$ injection: The Leading Edge, 16, 1300-1307.

Lee, D. S., Stevenson, V. M., Johnston, P. F., and Mullen, C. E., 1995 Time-lapse crosswell seismic tomography to characterize flow structure in the reservoir during thermal stimulation: Geophysics, 60, 660-666.

Luneburg, R. K., 1966. Mathematical Theory of Optics, University of California Press, Berkeley.

Maruyama, T., 1964, Statical elastic dislocations in an infinite and semi-infinite medium: Bull. Earthquake Prev. Res. Inst. Univ. Tokyo, 42 , 289-368.

Massonnet, D. and Feigl, K. L, 1998, Radar interferometry and its application to changes in the Earth's surface: Rev. Geophys., 36, 441-500.

Massonnet, D., T. Holzer, and H. Vadon, 1997, Land subsidence caused by East Mesa geothermal field, California, observed using SAR interferometry: Geophys. Research Letters., 24, 901-904.

Mossop, A., and P. Segall, 1999. Volume strain within the Geysers geothermal field: J. Geophys. Res., 104, 29113-29131. 
Masumoto, K., H., Tosaka, K. Kojimi, K. Itoh, and Y. Otsuka, 1995, New measuring system and high speed three dimensional inversion method for hydropulse tomography: in 8th International Congress on Rock Mechanics, edited by T. Fuji, 847-850, A. A. Balkema, Brookfield, Vt..

Murphy, H., Huang, C., Dash, Z., Zyvoloski, G., and White, A., 2004, Semianalytical solutions for fluid flow in rock joints with pressure-dependent openings: Water Resources Research, 40, W12506, doi:10.1029/2004WR003005.

Nur, A., 1989, Four-dimensional seismology and (true) direct detection of hydrocarbon: The petrophysical basis: The Leading Edge, 8, 30-36.

Obi, E-O. I., and Blunt, M., 2006, Streamline-based simulation of carbon dioxide storage in a North Sea aquifer: Water Resour. Res., 42, W03414, 1-13.

Paige, C. C., and Saunders, M. A., 1982, LSQR: An algorithm for sparse linear equations and sparse linear systems: ACM Trans. Math. Software, 8 , 195-209.

Paillet, F. L., 1993, Using borehole geophysics and cross-borehole flow testing to define connections between fracture zones in bedrock aquifers: J. Appl. Geophys., 30, 261-279.

Palmer, I. D., 1990, Uplifts and tilts at Earth's surface induced by pressure transients from hydraulic fractures: SPE Prod. Eng., 5, 324-332.

Parker, R. L., 1994, Geophysical inverse theory: Princeton University Press.

Press, W. H., S. A. Teukolsky, W. T. Vetterling, and B. P. Flannery, 1992, Numerical Recipes, Cambridge University Press, Cambridge.

Pruess, K., 2004, Numerical simulation of $\mathrm{CO}_{2}$ leakage from a geologic disposal reservoir, including transitions from super- to subcritical conditions, and boiling of liquid $\mathrm{CO}_{2}$ : SPE Journal, June, 237-248. 
Pruess, K., C. Oldenburg, and G. Moridis, 1999, TOUGH2 User's Guide, Version 2.0: LBNL Report, 43134, Berkeley.

Rice, J. R., and M. P. Cleary, 1976, Some basic stress diffusion solutions for fluidsaturated elastic porous media with compressible constituents: Rev. Geophys. Space Phys., 14, 227-241.

Rickett, J., L. Duranti, Hudson, T., Regel, B., and Hodgson, N., 2007, 4-D time strain and the seismic signature of geomechanical compaction at Genesis: The Leading Edge, 26 , 644-647.

Roste, T., Stovas, A., and Landro, M., 2006, Estimation of layer thickness and velocity changes using 4D prestack seismic data, Geophysics, 71, S219-S234.

Sasagawa, G. S., Crawford, W., Eiken, O., Nooner, S., Stenvold, T., Zumberge, M. A., 2003, A new sea-floor gravimeter, Geophysics, 68, 544-553.

Schmidt, D. A., and Burgmann, R., 2003, Time-dependent land uplift and subsidence in the Santa Clara valley, California, from a large interferometric synthetic aperture radar data set, J. Geophys. Res., 108, doi:10.1029/20002JB002267,4-1/4-13.

Segall, P., 1985, Stress and subsidence resulting from subsurface fluid withdrawal in the epicentral region of the 1983 Coalinga earthquake: J. Geophys. Res., 90, 6801-6816.

Sethian, J. A., 1996, Level Set Methods, Cambridge University Press.

Shapiro, S. A., Rothert, E., Rath, V., and Rindschwentner, J., 2002, Characterization of fluid transport properties of reservoirs using induced microseismicity, Geophysics, 67, 212-220.

Spiess, F. N., Chadwell, C. D., Hildebrand, J. A., Young, L. E., Purcell, G. H., and 
Dragert, H., 1998, Precise GPS/Acoustic positioning of seafloor reference points for tectonic studies: Earth Planet. Sci. Lett., 108, 101-112.

Stancliffe, R. P. W., and M. W. A. van der Kooij, 2001, The use of satellite-based radar interferometry to monitor production activity at the Cold Lake heavy oil field, Alberta, Canada: American Association of Petroleum Geologists Bulletin, 85, 781-793.

Staples, R., Ita, J., Burrell, R., and Nash, R., 2007, Monitoring pressure depletion and improving geomechanical models of the Shearwater Field using 4D seismic: The Leading Edge, 26 , 636-642.

Tura, A., Barker, T., Cattermile, P., Collins, C., Davis, J., Hatchell, P., Koster, K., Schutjens, P., and Willis, P., 2005, Monitoring primary depletion reservoirs using amplitudes and time shifts from high-repeat seismic surveys: The Leading Edge, 24, 1214-1221.

Tura, A., and Lumley, D. E., 1999, Estimating pressure and saturation changes from time-lapse AVO data: 61st Ann. Conf. Eur. Assoc. Geosci. Eng., Extended Abstracts, $1-38$.

Vasco, D. W., 2004a, Seismic imaging of reservoir flow properties: Time-lapse pressure changes: Geophysics, 69, 511-521.

Vasco, D. W., 2004b, Estimation of flow properties using surface deformation and head data: A trajectory-based approach: Water Resources Research, 40, doi:10.1029/2004WR003272, 1-14.

Vasco, D. W., Johnson, L. R., and Goldstein, N., 1988, Using surface deformation and strain observations to determine deformation at depth, with an application to Long Valley Caldera, California: J. Geophys. Res., 93 , 3232-3242. 
Vasco, D. W., Karasaki, K., and Myer, L., 1998. Inversion of surface tilt caused by fluid migration: J. of Geotech. and Geoenv. Eng., 124, 29-37 .

Vasco, D. W., Karasaki, K., and Doughty, C., 2000a, Using surface deformation to image reservoir dynamics: Geophysics, 65, 132- 147.

Vasco, D. W., K. Karasaki, and H. Keers, 2000b, Estimation of reservoir properties using transient pressure data: An asymptotic approach: Water Resour. Res., 36, 3447-3465.

Vasco, D. W., Karasaki, K. and Kishida, K., 2001. A coupled inversion of pressure and surface displacement: Water Resour. Res., 37, 3071-3089.

Vasco, D. W., Wicks, C., and Karasaki, K., 2002. Geodetic imaging: High resolution reservoir monitoring using satellite interferometry: Geophys. J. Int., 149, 555-571.

Vasco, D. W., and K. Karasaki, 2001, Inversion of pressure observations: an integral formulation: J. of Hydrology, 253, 27-40.

Vasco, D. W., and Finsterle, S., 2004, Numerical trajectory calculations for the efficient inversion of flow and tracer observations: Water Resour. Res., 40, W01507, $1-17$.

Vasco, D. W., and A. Ferretti, 2005, On the use of quasi-static deformation to understand reservoir fluid flow: Geophysics, 70, O13-O27.

Vesselinov, V. V., S. P. Neuman, and W. A. Illman, 2001, Three-dimensional numerical inversion of pneumatic cross-hole tests in unsaturated fractured tuff 2 . Equivalent parameters high-resolution stochastic imaging, and scale effects: Water Resour. Res., 37, 3001-3017.

Virieux, J., C. Flores-Luna, and D. Gibert, 1994, Asymptotic theory for diffusive electromagnetic imaging: Geophys. J. Int., 119, 857-868. 
Wang, R., and H.-J., Kuempel, 2003, Poroelasticity: Efficient modeling of strongly coupled, slow deformation processes in a multilayered half-space: Geophysics, 68, 705-717.

Wilt, M. J., Alumbaugh, D. L., Morrison, H. F., Becker, A., Lee, K. H., and DeszczPan, M., 1995, Crosswell electromagnetic tomography: System design considerations and field results: Geophysics, 60, 871-885.

Wright, C. A., 1998, Tiltmeter fracture mapping: From the surface and now downhole: Petro. Eng. Int., January, 50-63.

Wright, C. A., Davis, E. J., Minner, W. A., Ward, J. F., Weijers, L., Schell, E. J., and Hunter, S. P., 1998, Surface tiltmeter fracture mapping reaches new depths 10,000 feet and beyond?: SPE, 39919, April.

Wu, Y.-, S. and K. Pruess, 2000, Integral solutions for transient fluid flow through a porous medium with pressure-dependent permeability: Int. J. Rock Mech. and Mining Sci., 37, 51-61.

Yeh, T.-C. J. and S. Liu, 2000, Hydraulic tomography: Development of a new aquifer test method: Water Resour. Res., 36, 2095-2105. 


\section{APPENDIX: DEFORMATION IN THE ELASTIC OVERBURDEN}

Due to the porosity changes, induced by fluid pressure build-up or decay in a reservoir or aquifer, there is a corresponding volume change. The volume change induces deformation in the surrounding material which is transmitted through the subsurface. In this Appendix we note how such deformation may be used to estimate reservoir pressure changes.

\section{Estimation of volume change}

The first step in the estimation procedure is to use the deformation data to infer variations in pressure. This aspect has already been discussed thoroughly by Vasco et al. (2001). We will only state the final result, a system of linear equations relating volume changes within each grid block of a reservoir model $\mathbf{v}_{f}(t)$ to pressure changes $\delta \mathbf{p}(t)$ and deformation observations $\mathbf{u}(t)$

$$
\left[\begin{array}{c}
\mathbf{u}(t) \\
\delta \mathbf{p}(t)
\end{array}\right]=\left[\begin{array}{c}
\boldsymbol{\Upsilon} \\
\boldsymbol{\Pi}
\end{array}\right] \mathbf{v}_{f}(t)
$$

where the coefficients of the matrices $\Upsilon$ and $\boldsymbol{\Pi}$ are given by

$$
\Upsilon_{i j k}=\int_{V_{j}} g_{i}\left(\mathbf{x}_{k}, \mathbf{y}\right) d V
$$

for observation point $\mathbf{x}_{k}$, deformation components $i$ and reservoir grid block $j$, and

$$
\Pi_{i j}=C_{e}^{-1}\left[\delta_{j k}-\frac{B}{\rho} \int_{V_{j}} G_{i i}\left(\mathbf{x}_{k}, \mathbf{y}\right) d V\right]
$$

respectively, where $B$ is Skempton's pore pressure coefficient (Rice and Cleary 1976, Wang and Kuempel 2003) and $\rho$ is the density. The integrand in (A2), $g_{i}\left(\mathbf{x}_{k}, \mathbf{y}\right)$, is the Green's function which is the point response of the elastic medium at location $\mathbf{x}_{k}$. As such, it is medium-dependent, as discussed in Vasco et al. (2000a). The integral is evaluated over the volume of the $j$-th grid block, $V_{j}$. The kernel $G_{i i}$ in the integral 
in (A3) is constructed from the Green's function solution $g_{i}$, as described in Segall (1985)

$$
G_{i i}=(2 \mu+\lambda) \frac{\partial g_{i}}{\partial x_{i}}
$$

where there is no summation over the index $i$. Note that one could allow the poroe-

lastic coefficients $C_{e}^{-1}$ to vary over the aquifer or reservoir, assuming that such information is known. The system of equations (A1) is typically unstable with respect to perturbations, numerical or otherwise, and some form of regularization is required. Thus, the system is solved using a regularized least squares algorithm, as discussed in Vasco et al. (2000b, 2001).

\section{Mapping estimates of volume change into changes in pressure and overburden stress}

Once estimates of the volume change within the reservoir are available, by solving the system of equations (A1), we map them into fluid pressure and overburden stress changes. The mapping is described in more detail in Vasco et al. (2001) and is based on the work of Segall (1985). The fluid pressure change is given by

$$
\delta p(\mathbf{x}, t)=C_{\nu} v_{f}(\mathbf{x}, t)-\frac{B^{2}}{3 \rho} \int_{V} G_{i i}(\mathbf{x}, \mathbf{y}) v_{f}(\mathbf{y}, t) d V
$$

where

$$
C_{\nu}=\frac{B^{2}}{\rho}\left[\mu \frac{2}{3} \frac{\left(1+\nu_{d}\right)\left(1+\nu_{u}\right)}{\left(\nu_{u}-\nu_{d}\right)}-3 K_{u}\right],
$$

$\nu_{u}$ and $\nu_{d}$ are the undrained and drained Poisson's ratios (Rice and Cleary 1976), $K_{u}$ is the undrained bulk modulus, and the diagonal components of the overburden stress are given by

$$
\sigma_{i i}=\frac{B}{\rho} \int_{V} G_{i i}(\mathbf{x}, \mathbf{y}) v_{f}(\mathbf{y}, t) d V-\frac{B K_{u}}{\rho} v_{f}(\mathbf{x}, t) .
$$

Note that all of the mappings are linear, as is the inverse problem for volume change, equation (A1). The second term in equation (A5) accounts for propagation of stress within the overburden in the calculation of reservoir pressure change. 


\section{FIGURE CAPTIONS}

Figure 1. Spatial permeability variation used to generate a set of surface displacement values. The gray scale denotes the logarithm of the permeability variations within the reservoir model.

Figure 2. Four snapshots of range change [24, 128, 408, and 842 days] induced by pressure change within the reservoir. The filled squares indicate a decrease in range, the distance between the point on the surface and a hypothetical point in space. The reference square at the top of each panel signifies a change of $1.00 \mathrm{~cm}$.

Figure 3. Estimates of pressure change within the reservoir resulting from an inversion of the range change values in Figure 2. The gray scale indicates changes in pressure from the background values. The injection well is indicated by the unfilled star in each panel.

Figure 4. Time derivatives of the estimates of pressure change. The derivatives are normalized by the peak value attained in each grid block.

Figure 5. The solid squares denote phase or arrival time estimates used in the inversion for permeability. The solid curves represent the trajectories used in the inversion. The trajectories are calculated from the phase field, as indicated in equation (28).

Figure 6. Logarithm of the permeability multipliers resulting from the inversion of the phase values in Figure 6. The permeability estimates are obtained by solving the linear system of equations defined by the necessary equations for a minimum of $R$ in equation (31).

Figure 7. Observed deviation of the travel times from those predicted by a uniform permeability model, the background model. The observed deviations are plotted against the deviations predicted by the inversion result. For perfect agreement, the observed and predicted deviations would plot along the $45^{\circ}$ line shown in the figure. 
Figure 8. Range velocities estimated from scenes associated with two different tracks (65 and 294), for two satellite passes. The dark shades indicate a range decrease, due to uplift from $\mathrm{CO}_{2}$ injection at depth. The surface projection of the injection well is indicated by the solid lines in each panel.

Figure 9. Range change estimates obtained by re-sampling the values for Tracks 65 and 294, averaging values using a moving window, and stacking the values for the two tracks. The surface projection of the injection well is indicated by the solid lines in each panel.

Figure 10 Pressure estimates resulting from an inversion of the range change data shown in Figure 9. The gray scale indicates pressures in MPa, with dark tones indicating a pressure increase. The surface projection of the injection well is indicated by the solid lines in each panel.

Figure 11. The normalized time derivative calculated from the pressure estimates. The derivative is calculated for the pressure field after 305 days of injection. The derivative values have been normalized by the peak pressure in each grid block.

Figure 12a. The arrival time of the pressure transient, calculated from the pressure derivative estimates.

Figure 12b. The solid squares denote phase or arrival time estimates used in the inversion for permeability. The solid lines represent the trajectories used in the inversion. The trajectories are calculated from the phase field, as indicated in equation (28).

Figure 13. Logarithm of the permeability multipliers resulting from the inversion of the phase values in Figure 12b. The surface projection of the injection well is indicated by the solid line. The surface projection of fault, inferred from seismic data, is indicated by the dashed line.

Figure 14. Observed deviation of the travel times from those predicted by a uniform permeability model, the background model. The observed deviations are plotted against the deviations predicted by the inversion result. For perfect agreement, the 
observed and predicted deviations would plot along the $45^{\circ}$ line shown in the figure.

Figure 15. (Upper panel) Phase changes associated with an inversion of range change data from track 65. (Lower panel) Phase changes associated with an inversion of range change data from track 294.

Figure 16. (Upper panel) Permeability deviations from the uniform background model obtained from an inversion of the phase changes associated with Track 65, [see Figure 15]. (Lower panel) (Upper panel) Permeability deviations from the uniform background model obtained from an inversion of the phase changes associated with Track 294 . 


\section{FIGURES}




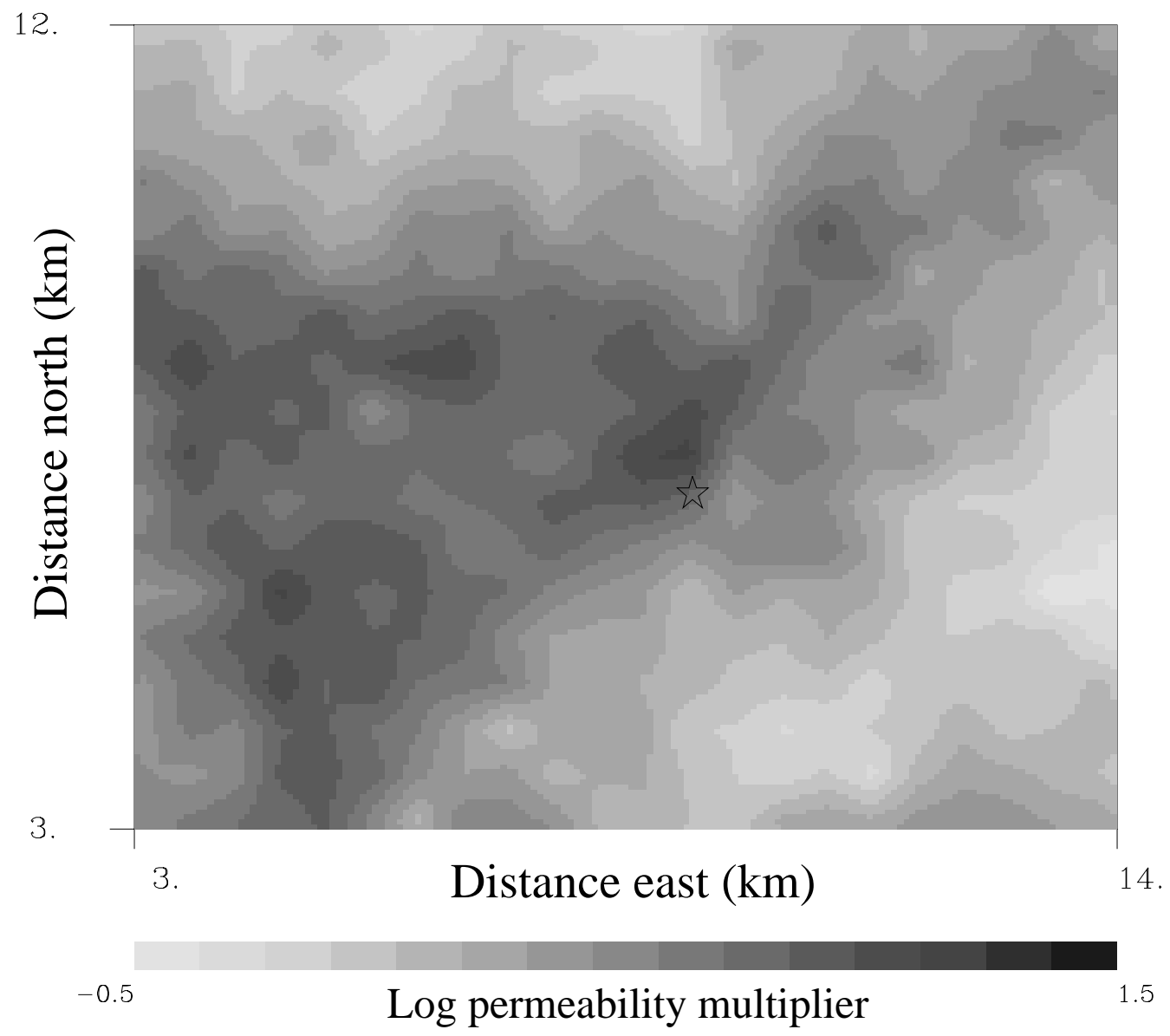

Figure 1. 


\section{Days}

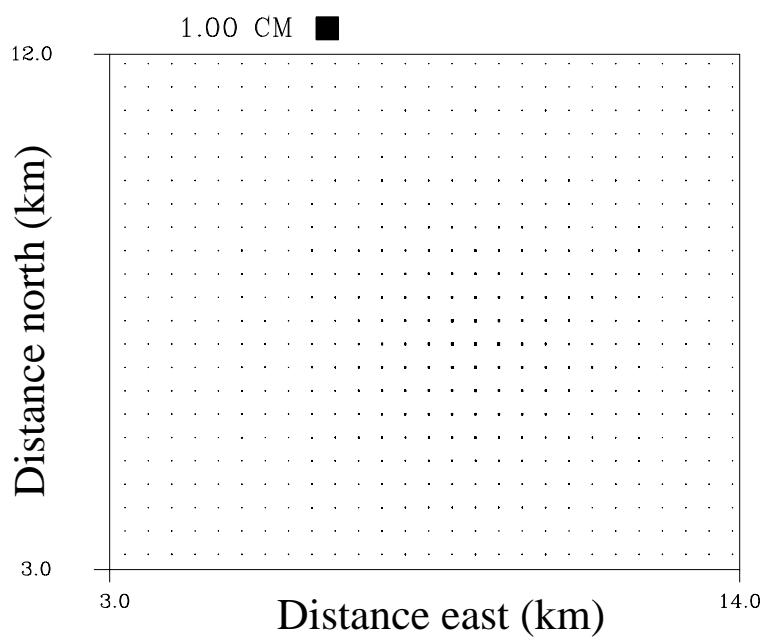

408 Days

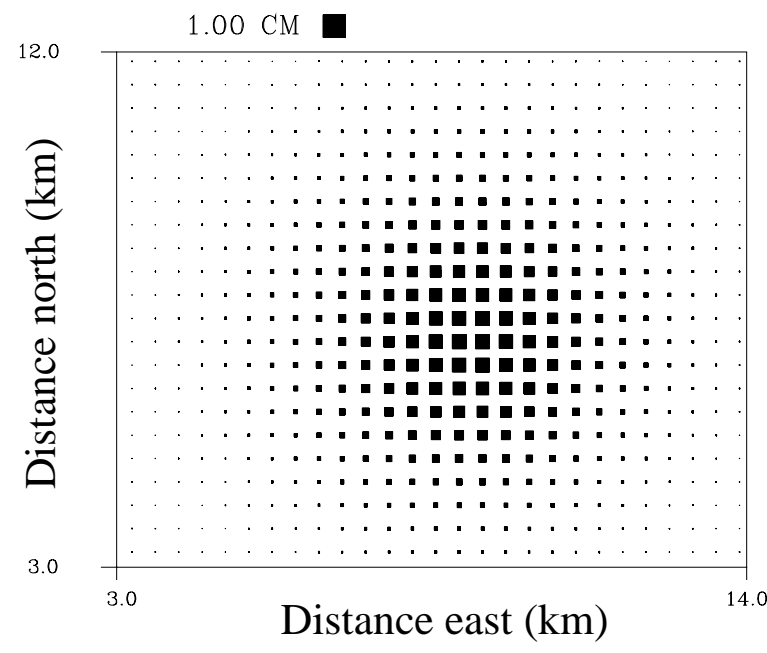

128 Days
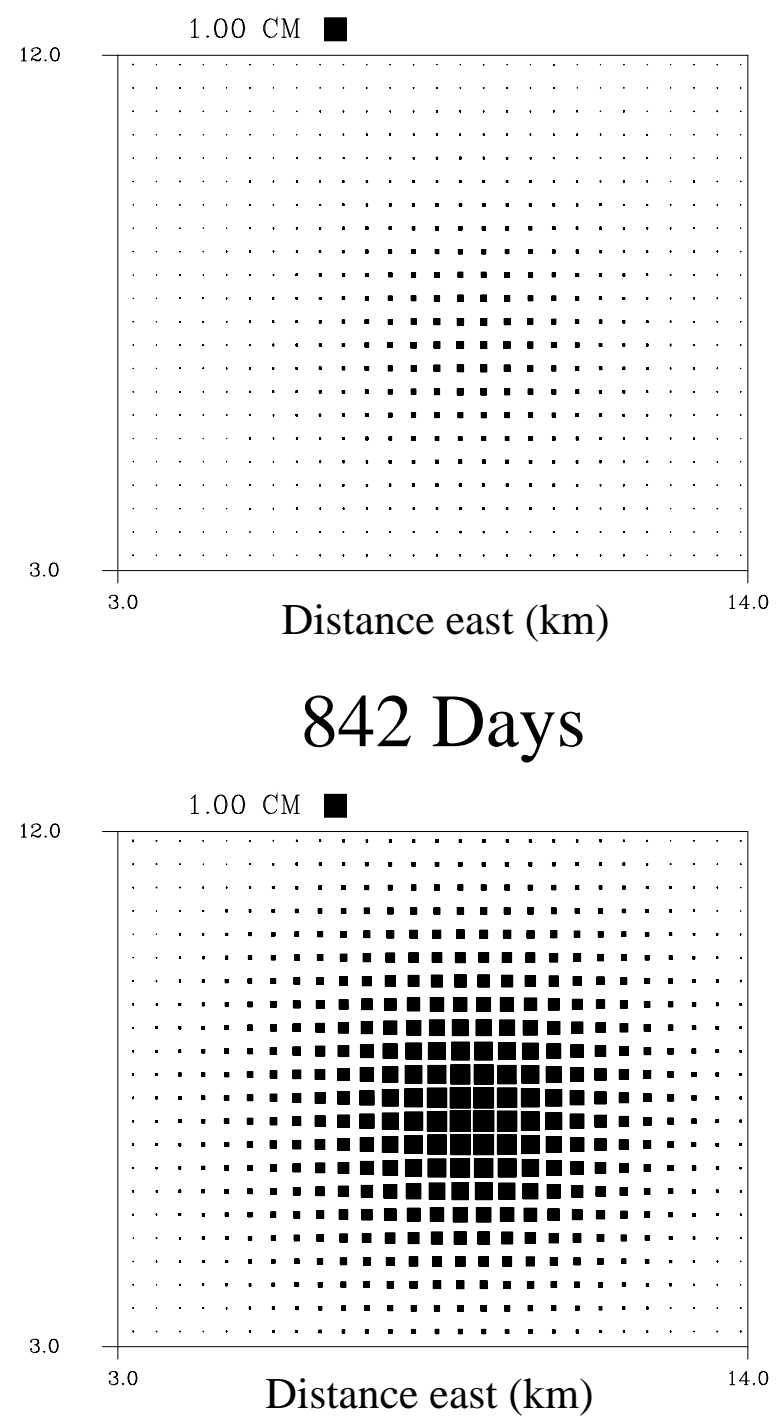

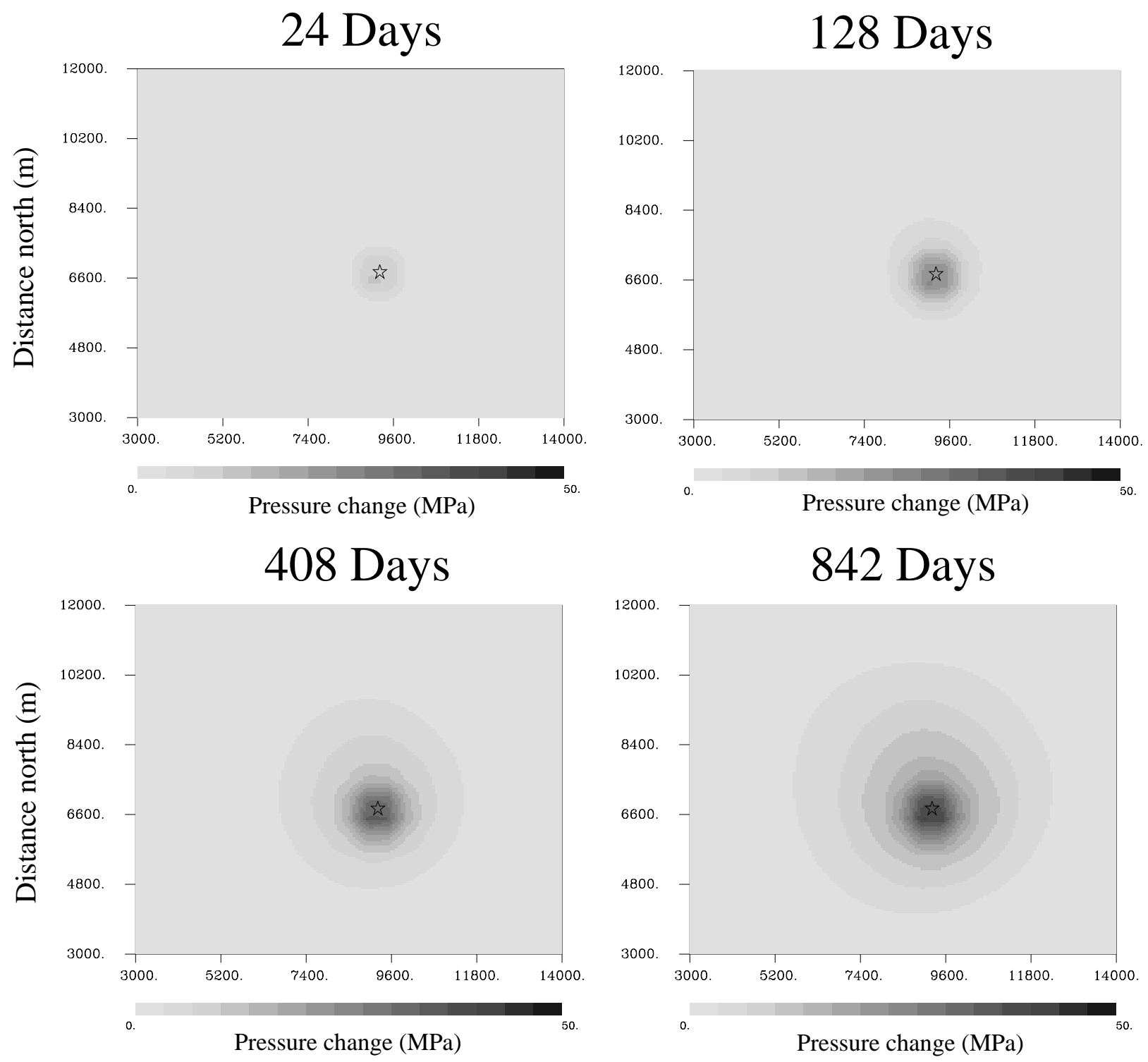

Figure 3. 

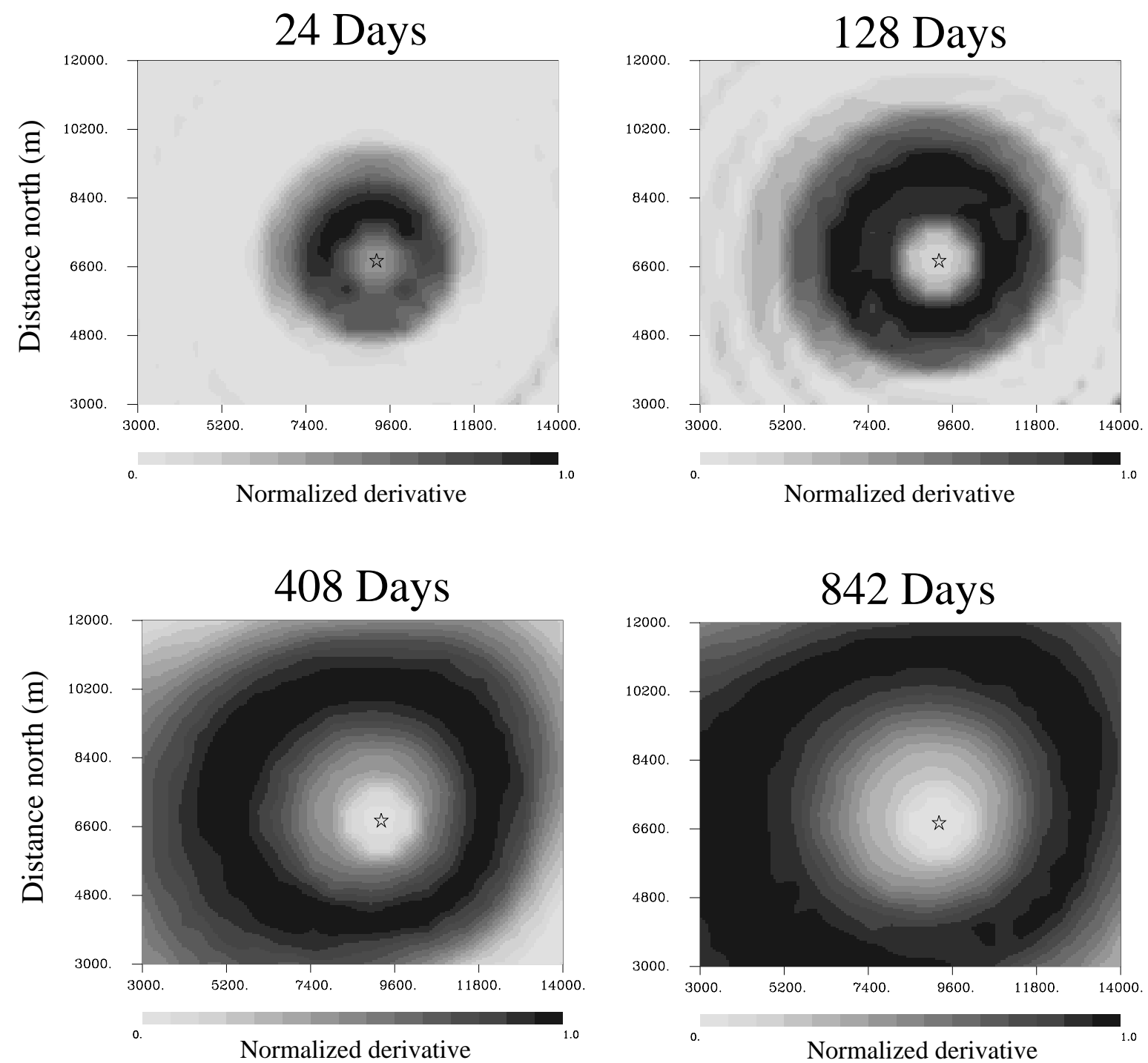

Figure 4 . 


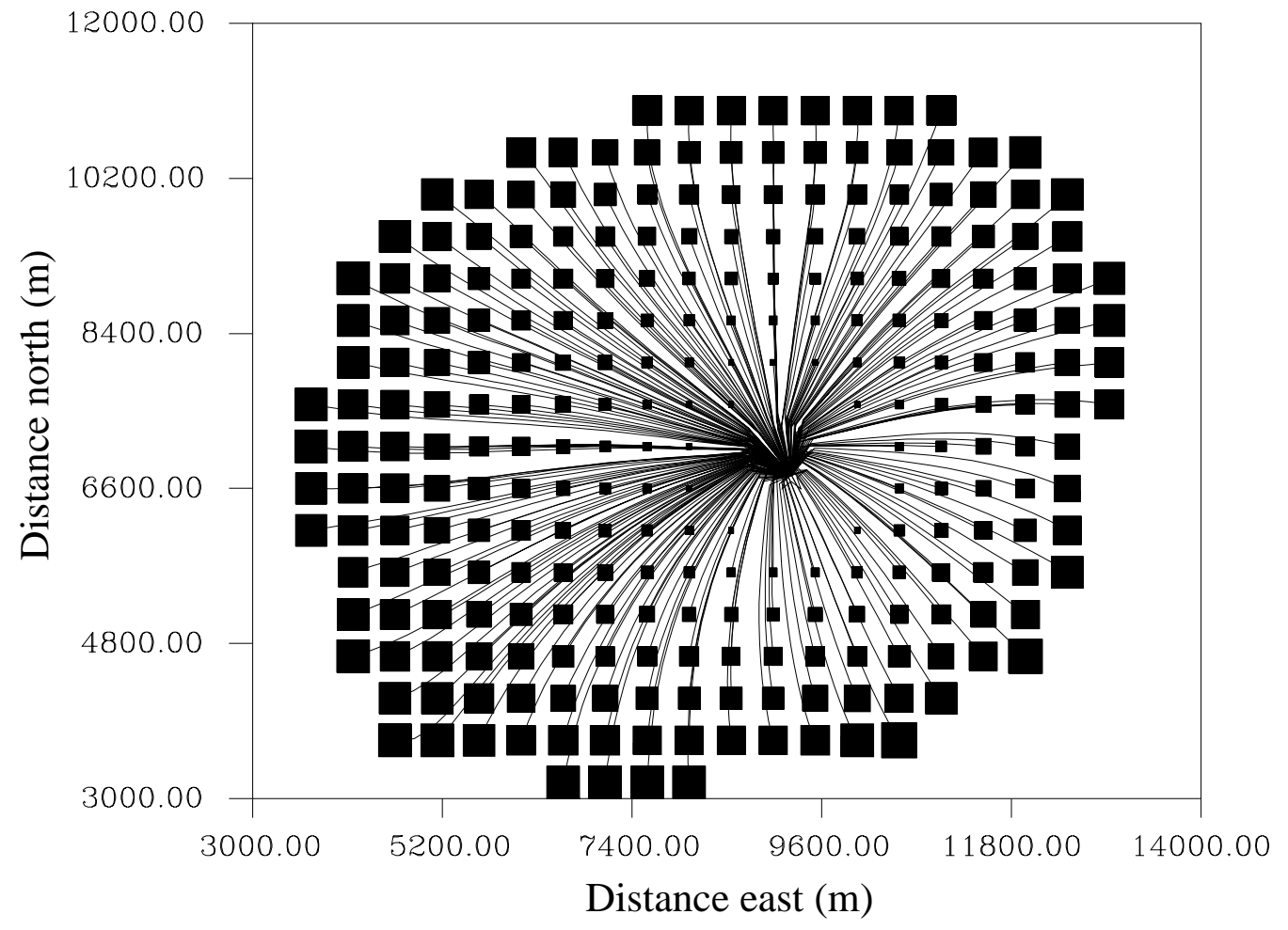

Figure 5 . 


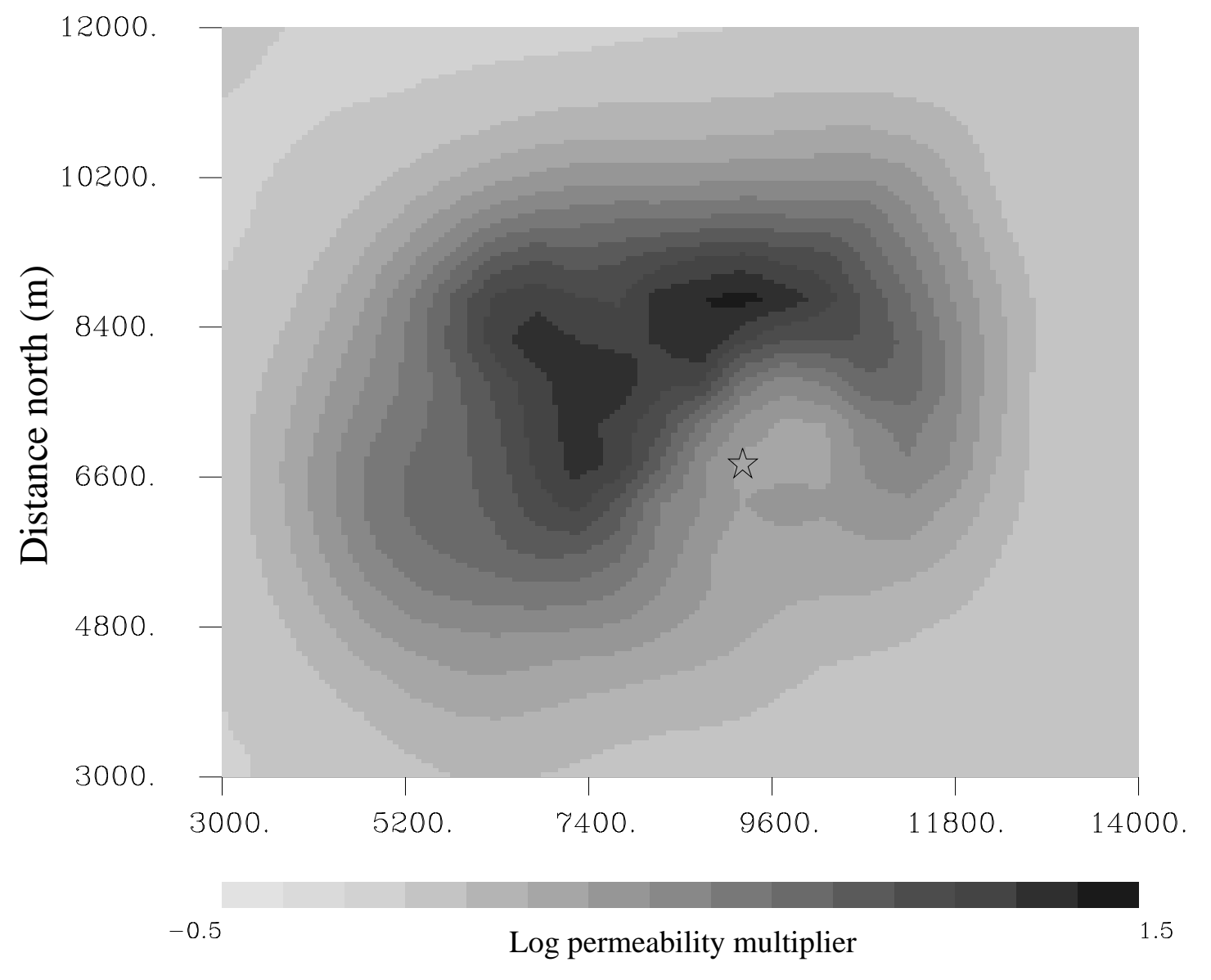

Figure 6. 


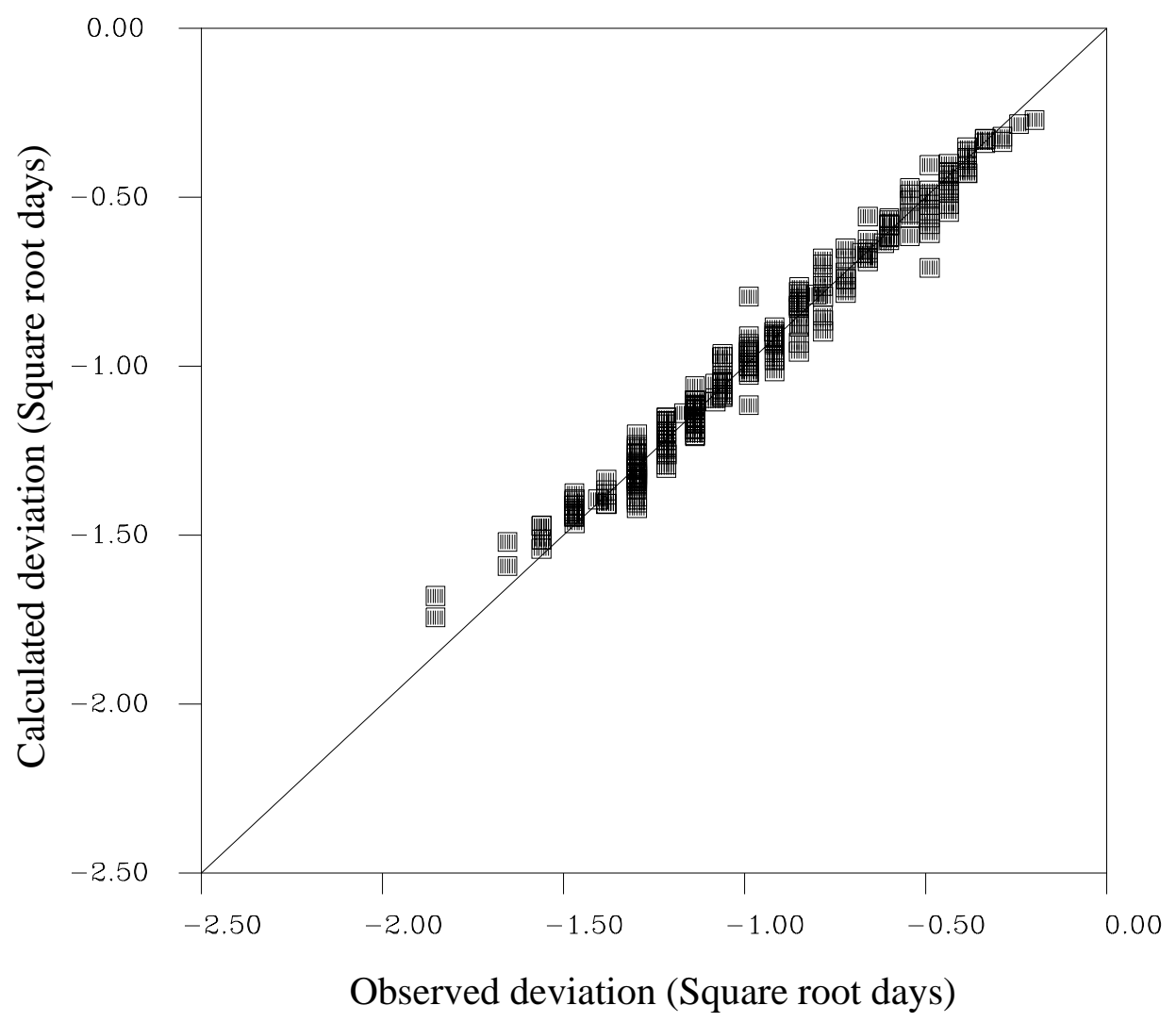

Figure 7 . 

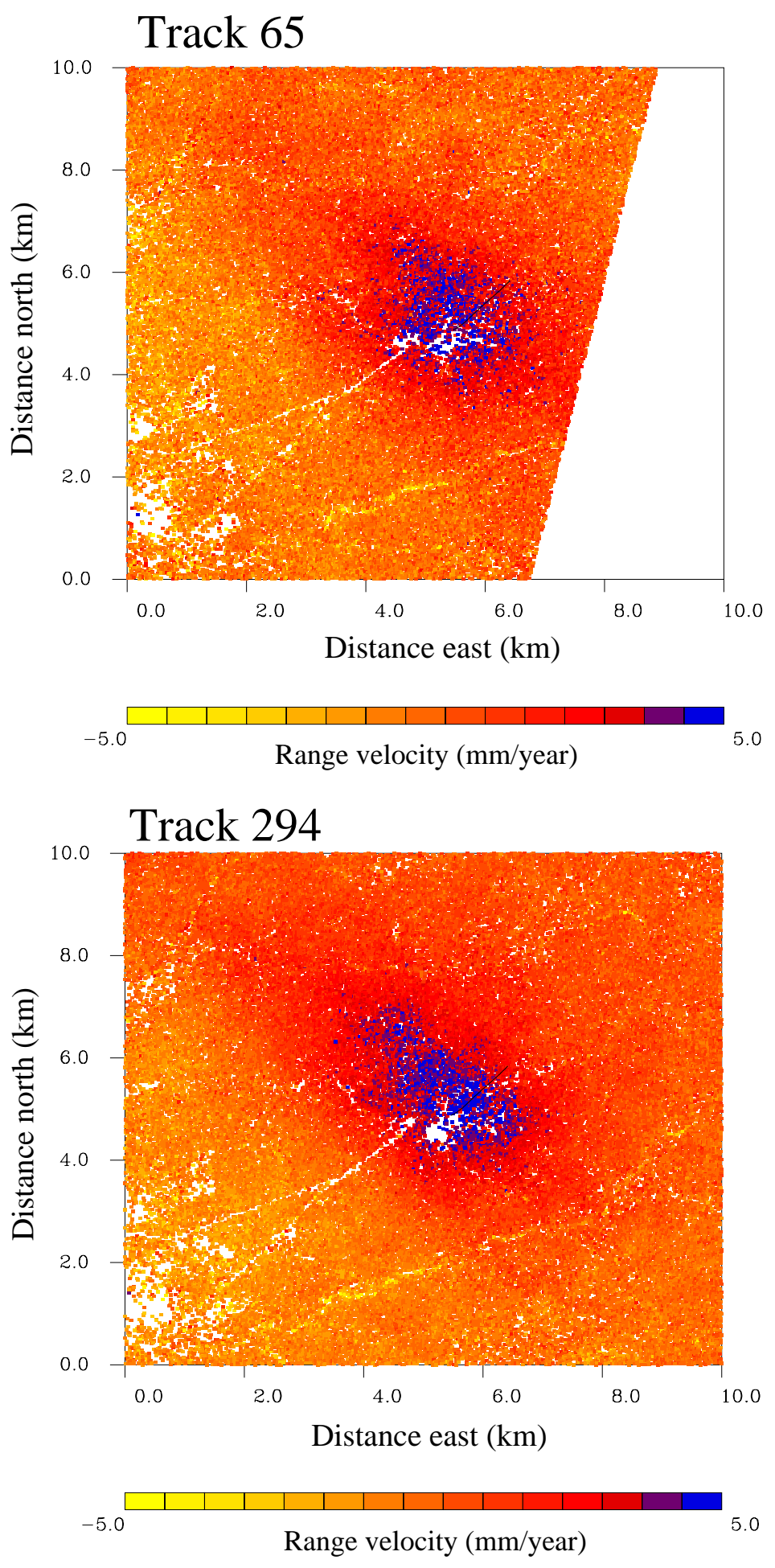

Figure 8. 

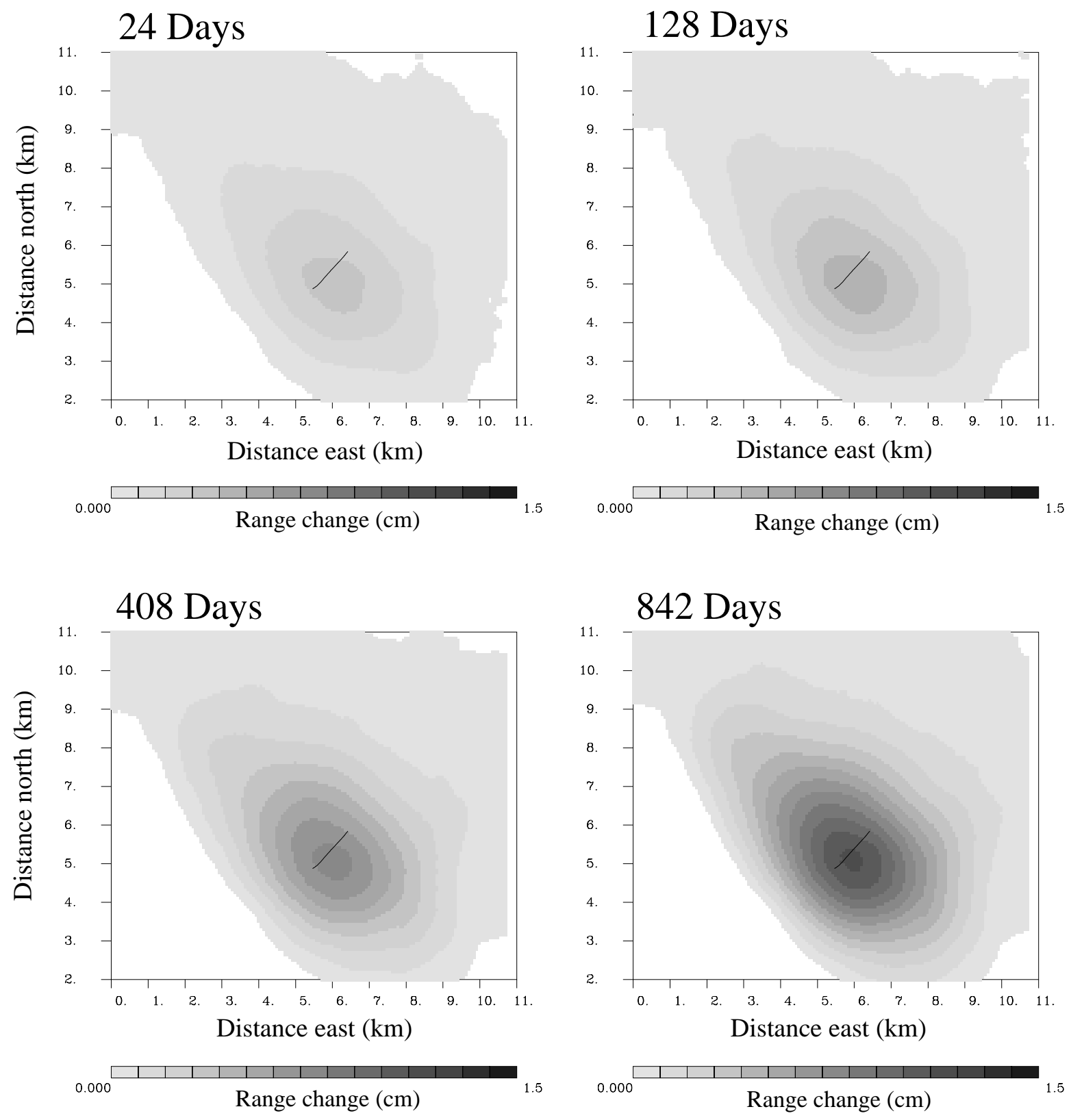

Figure 9. 


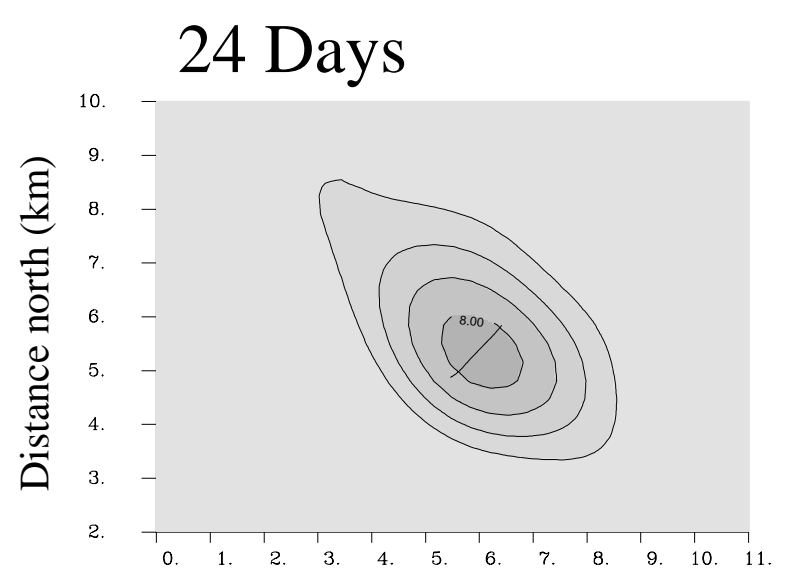

o. $\quad$ Pressure change (MPa)

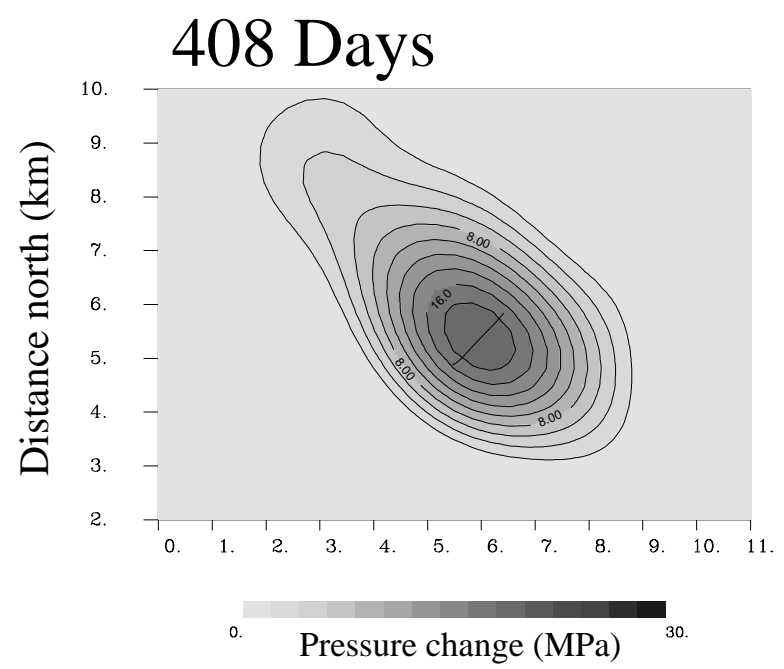

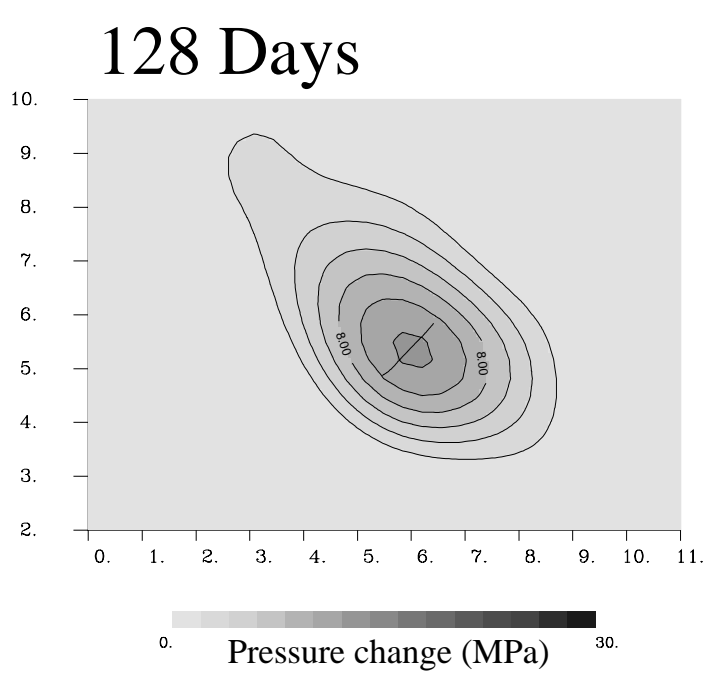

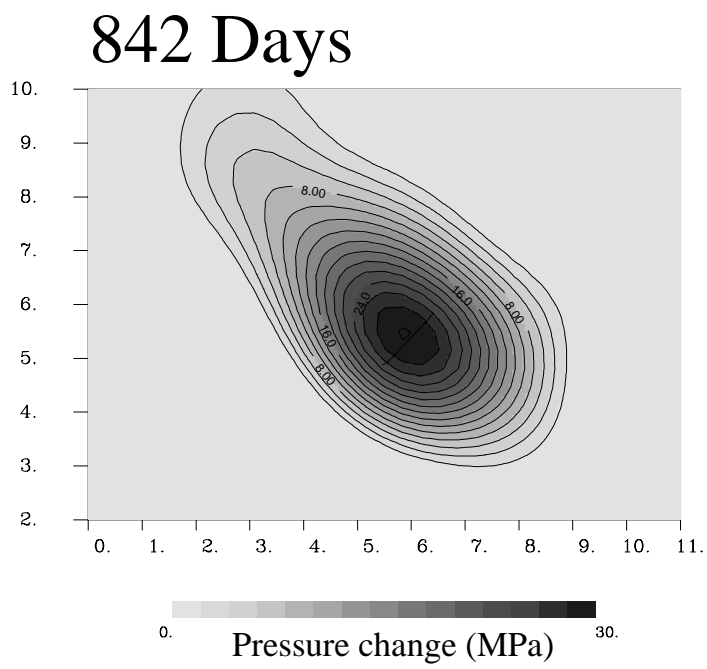

Figure 10. 


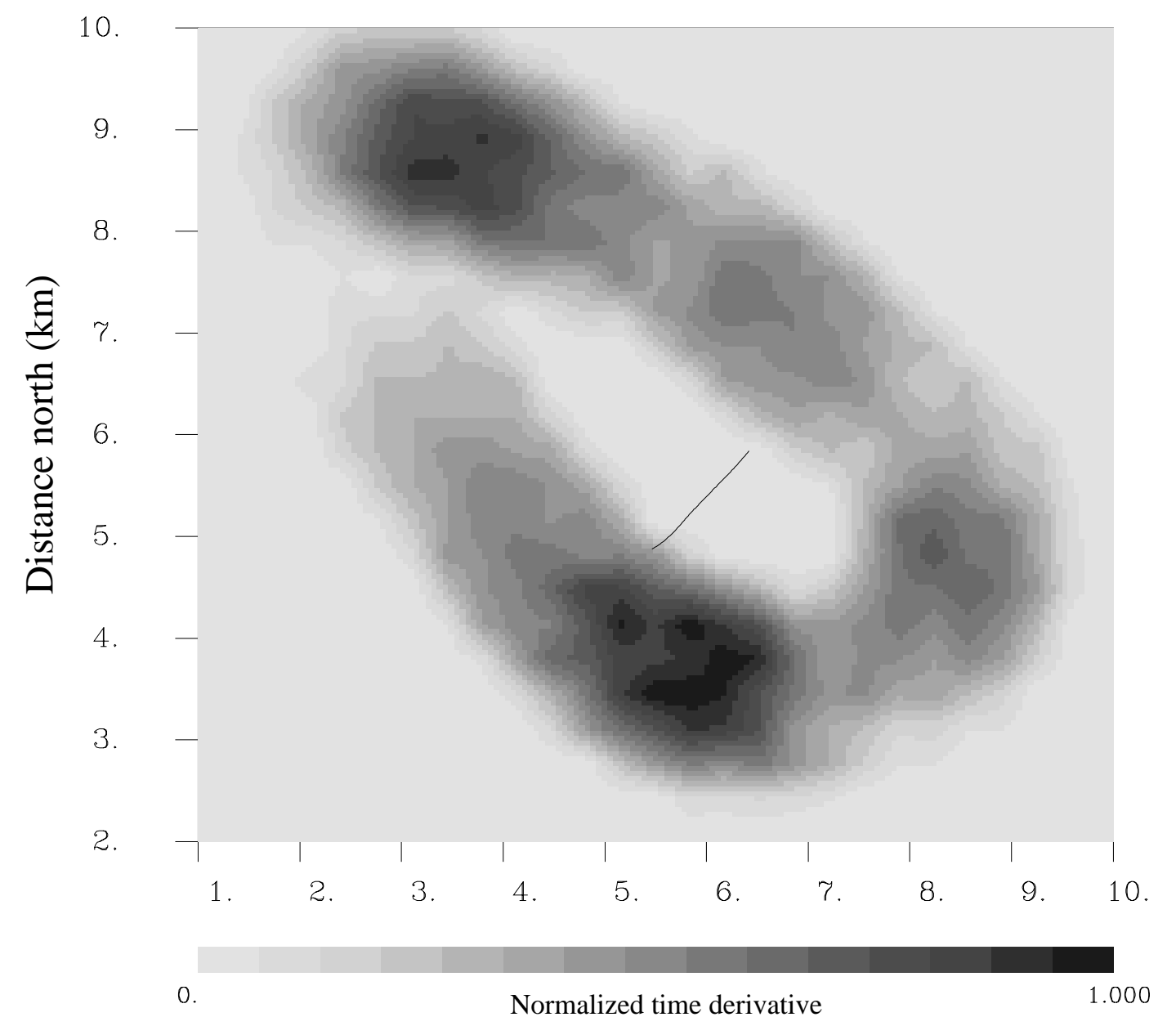

Figure 11. 


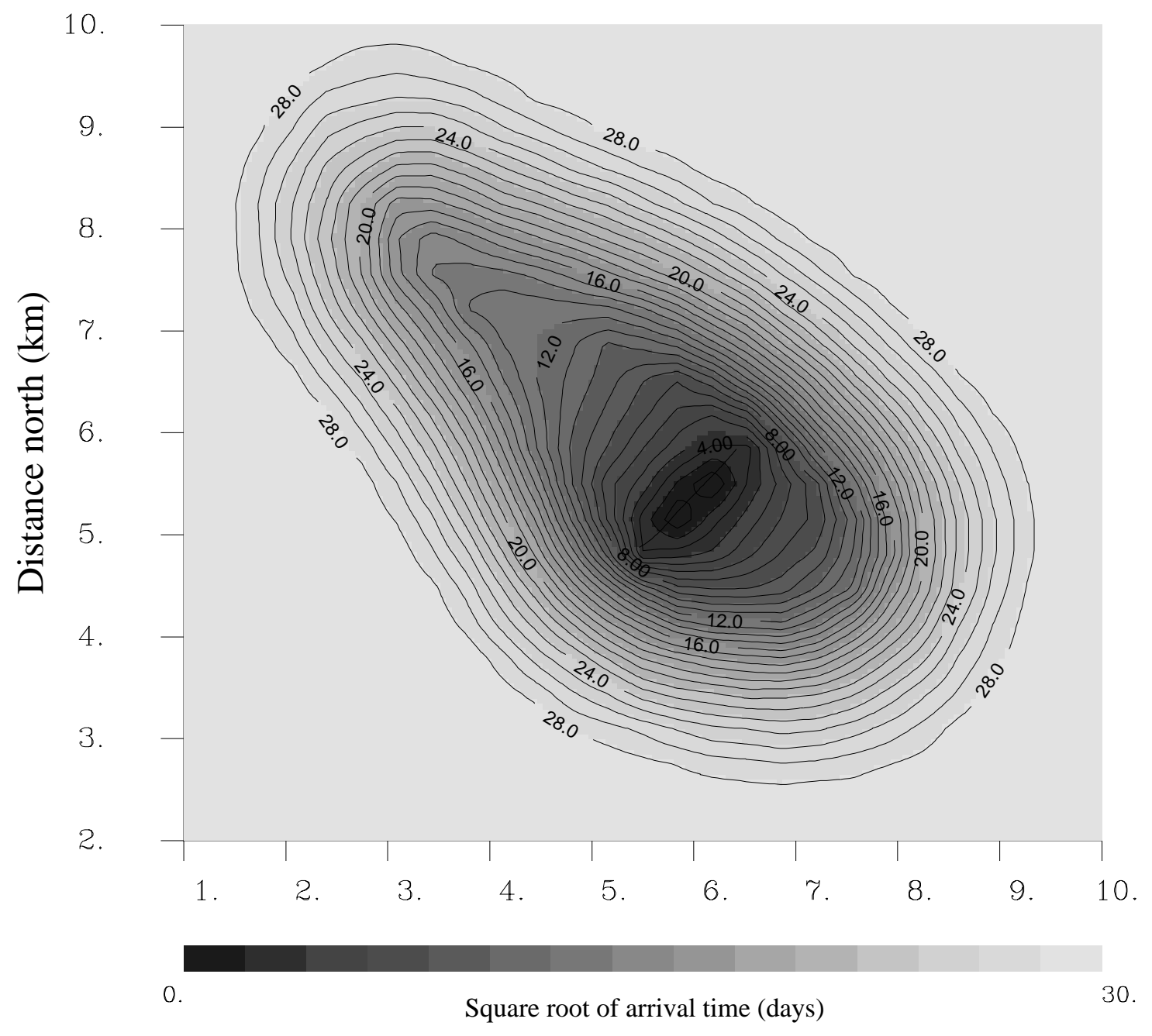

Figure $12 \mathrm{a}$. 


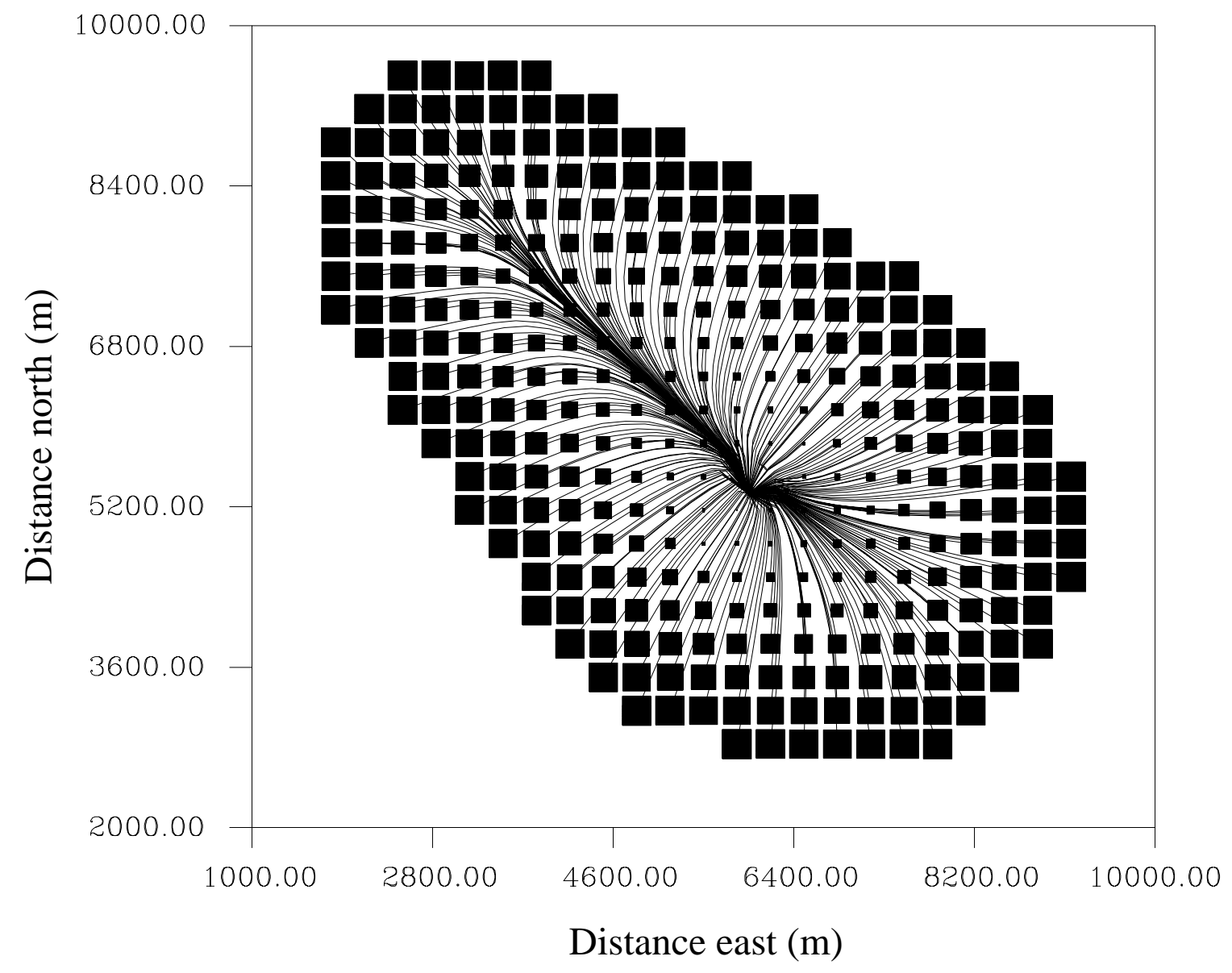

Figure 12b. 


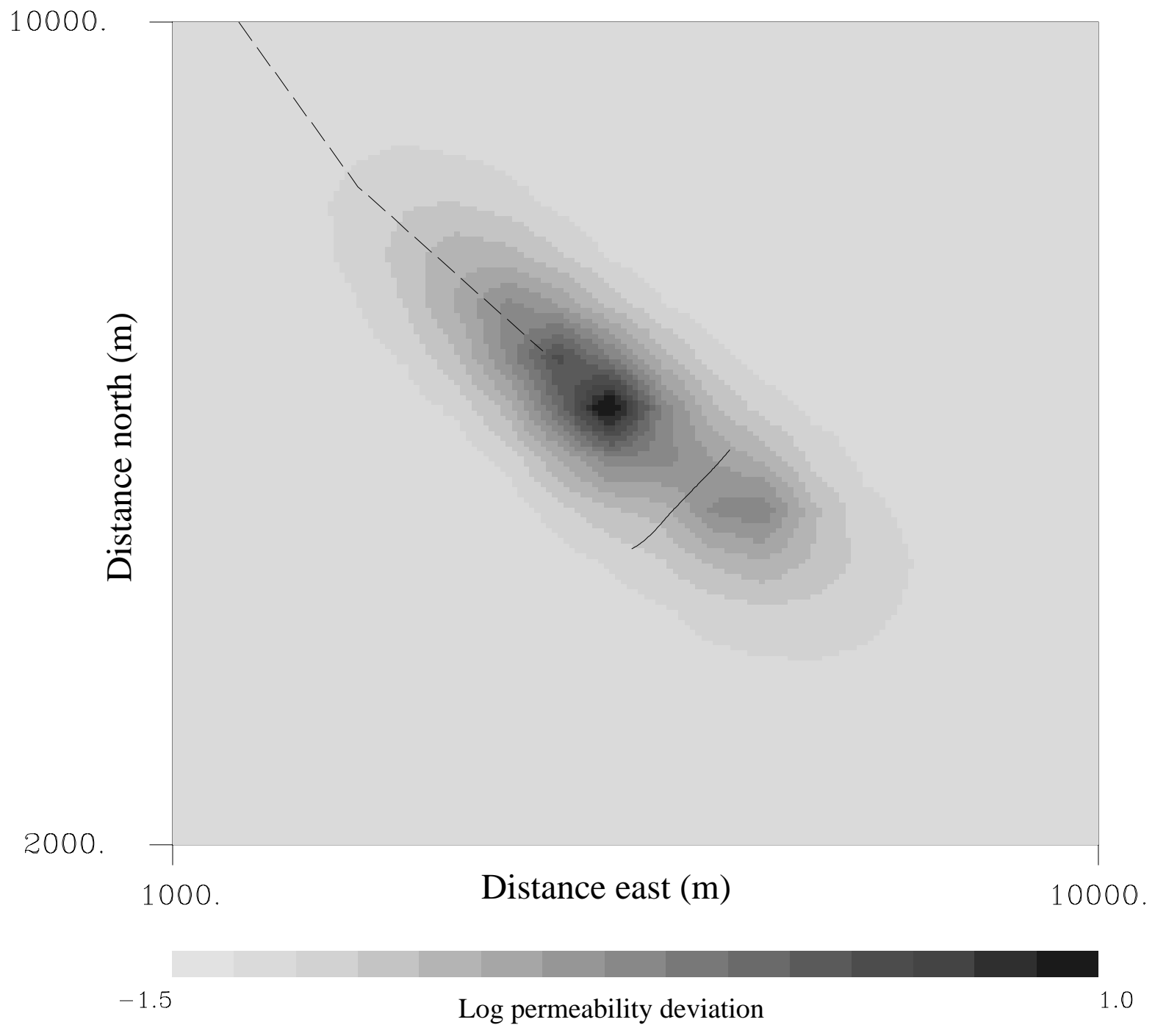

Figure 13. 


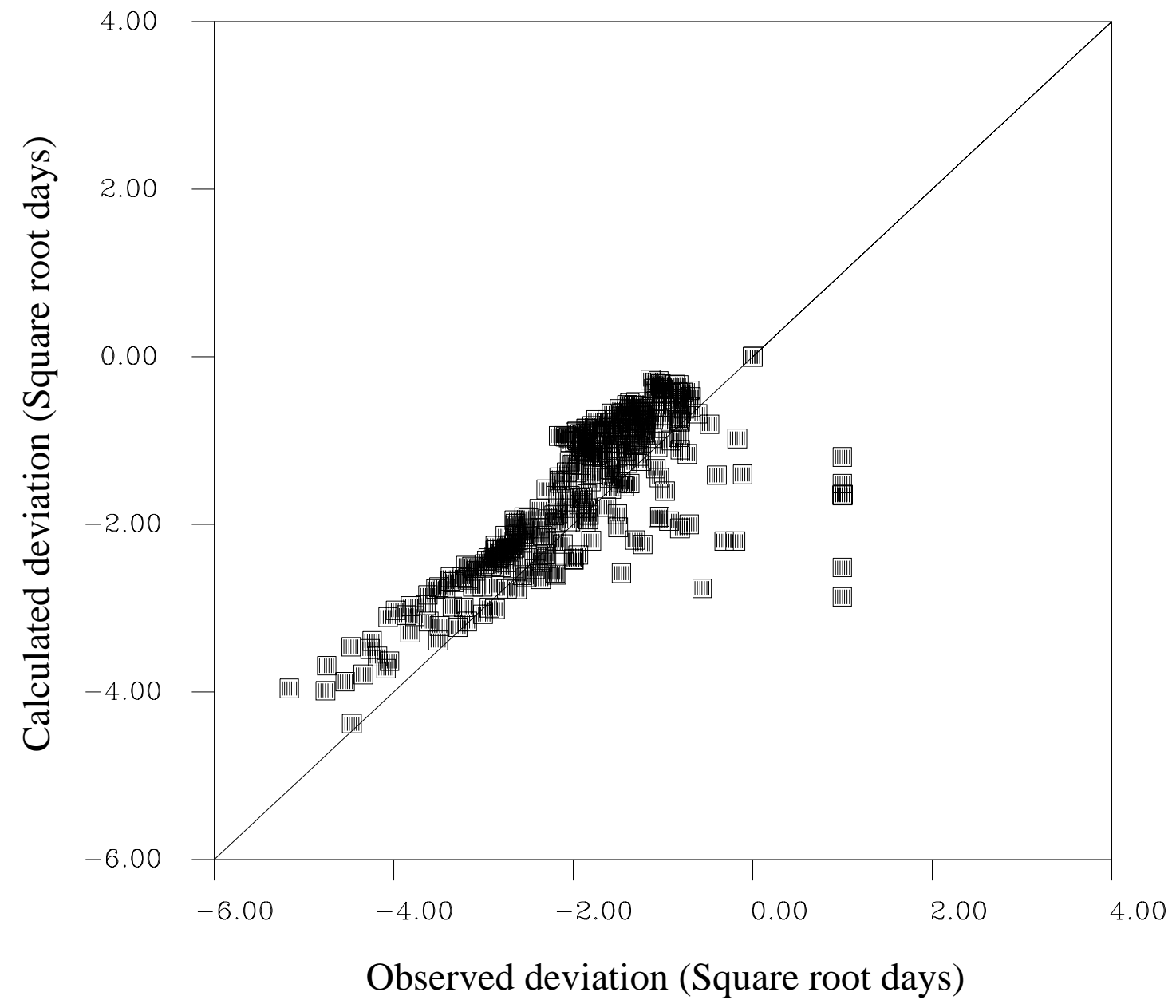

Figure 14. 

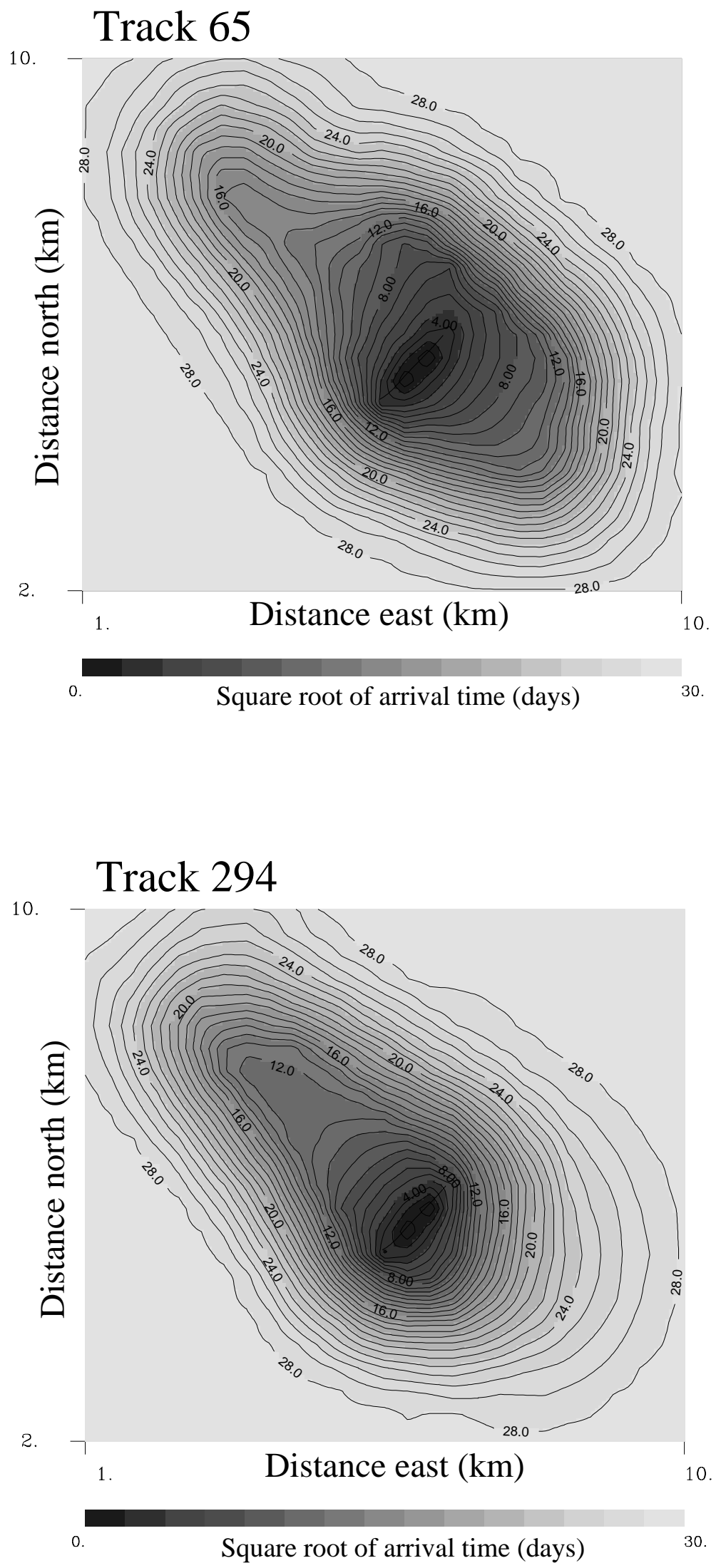

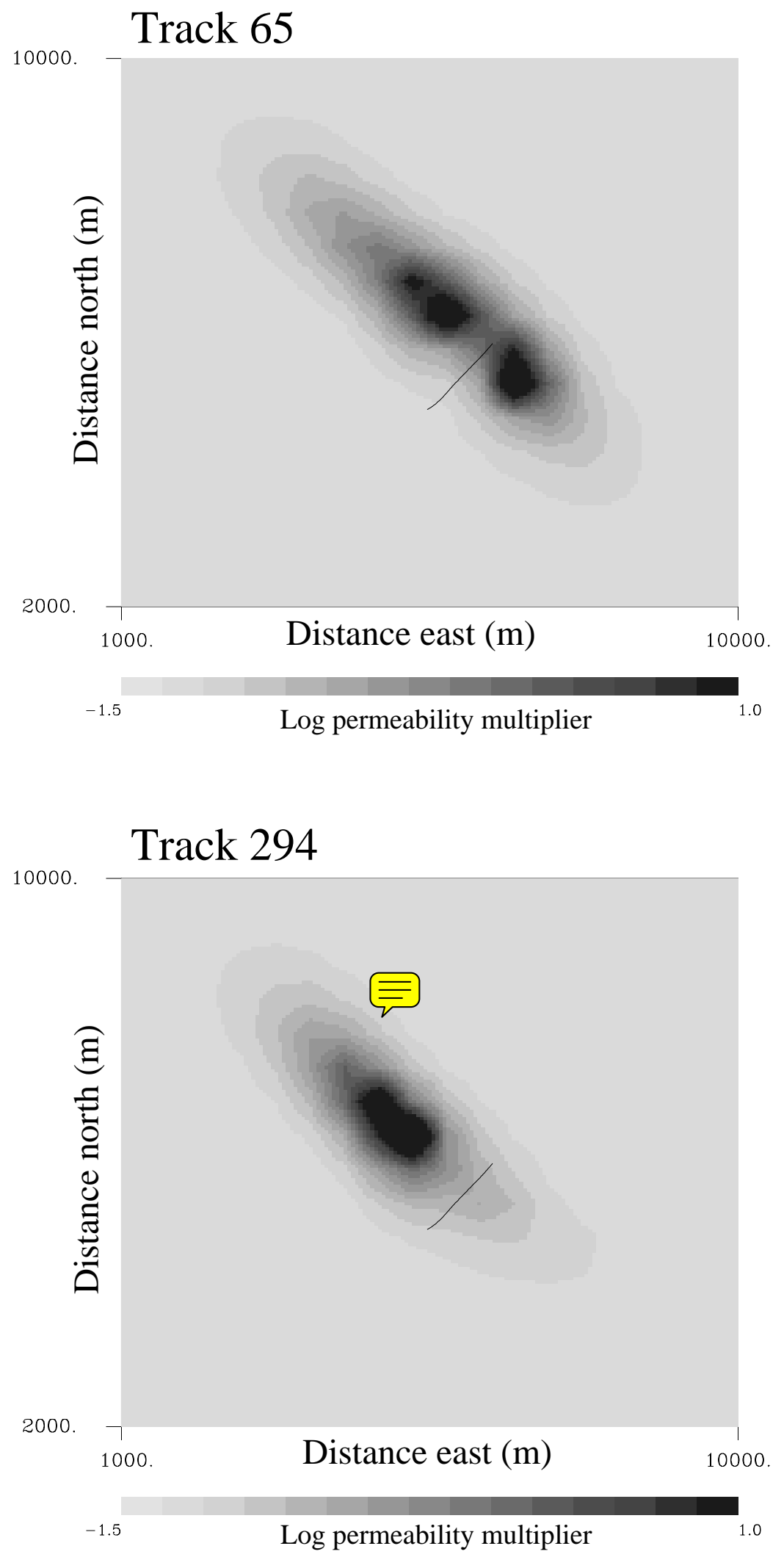

Figure 16. 